\title{
Finite and infinite block Petrov-Galerkin method for cracks in functionally graded materials
}

\author{
Y. $\mathrm{Li}^{1,2, *}, \mathrm{~J} . \mathrm{Li}^{3}$ and P.H. Wen ${ }^{4}$ \\ ${ }^{1}$ School of Mechanics and Engineering, Southwest Jiaotong University, Chengdu, China \\ ${ }^{2}$ Department of mathematics, City University of Hong Kong, Hong Kong SAR \\ ${ }^{3}$ School of Mathematics, Changsha University of Science \& Technology, Hunan, China \\ ${ }^{4}$ School of Engineering and Materials Science, Queen Mary University of London, London, UK
}

\begin{abstract}
This paper presents a novel weak-form block Petrov-Galerkin method (BPGM) for linear elastic and crack problems in functionally graded materials with bounded and unbounded problem domains. The main idea of this approach is to combine the meshless local Petrov-Galerkin method with block method. Once the problem domain is discretized into several sub-regions, named blocks, which can be mapped into normalized square domains. The weak-form Petrov-Galerkin method and polynomial series of interpolations are employed in each block. The computational efficiency is rigorously examined against the strong-form finite block method, the finite element technique and meshless approaches. Numerical results demonstrate that the BPGM possess the following important properties: (1) only a few blocks are required for calculating problems in unbounded regions which saves tedious work of meshing; (2) the displacements and stresses are continuous at the interfaces of neighboring blocks; (3) due to the use of weak formulation, the continuity requirements of the approximation functions are reduced and numerical solutions are stable; (4) because of using Lagrange polynomial interpolation, highly accurate results can be obtained with a small amount of nodes in each block.

Keywords: weak-form Petrov-Galerkin method, infinite block, meshless technique, polynomial interpolation, functionally graded materials, continuous displacements and stresses

*Corresponding author

Emails: liyan001de@gmail.com
\end{abstract}




\section{Introduction}

Many engineering problems can be modeled as an extension of either finite or semi-infinite regions. The analytical solutions of these models are often difficult to obtain due to the complexity of material properties and irregular geometries. Numerical techniques are developed to deal with these problems which are modeled by partial differential equations (PDEs), among which the widely used approaches are finite difference method (FDM) [1], finite element method (FEM) [2] and boundary element method (BEM) [3]. The FDM and FEM are mesh dependent and become computationally time demanding on generation of high quality meshes for efficient solutions. The use of fundamental solutions or Green's functions in BEM provides more accurate and efficient approximation. However, it is usually difficult to obtain the fundamental solutions in closed forms, especially for nonhomogeneous and anisotropic materials. In recent decades, meshless techniques were developed to solve practical problems and had drawn the attention of many investigators, including the smooth particle hydrodynamics method [4] , the diffuse element method [5], the element-free Galerkin method (EFG) [6] and the reproducing kernel particle method [7]. Based on the weak Petrov-Galerkin formulation, Atluri and his co-workers developed the meshless local Petrov-Galerkin method (MLPG) with moving least square approximation for problems in arbitrary geometries [8]. Sladek et al. [9] introduced the local boundary integral equation method for boundary value problems in anisotropic non-homogeneous media. Among these meshless approaches, the radial basis functions (RBFs) method [10] was a truly meshless collocation method using in particular the multiquadric shape function [11-12] for superior spectral convergence. However, it suffered from ill-conditioning problem. For large scale problems, the localized radial basis function method was developed and had been successfully applied to solve diffusion and convection-diffusion problems by Sarler et al. [13-14]. Liu et al. developed the radial point interpolation method based on a predefined background mesh to deal with various mechanical problems [15-16]. Recently, the combination of the finite integration method with RBFs [17-18] was developed to deal with stiff 
problems.

The conventional approach to solve problems in unbound field is FEM which truncates the infinite boundary at a finite location in the far-field. This approach consumes a lot of computation for domain discritization. In some cases, analytical solutions could be obtained by considering a relatively small and finite region and setting the state in remote field to be as simple as possible. Zienkiewicz [19] and Brebbia [20] et al. combined the BEM and the FEM and provided appropriate boundary conditions at the truncated boundary. This approach was, however, limited by the explicit expression of fundamental solutions. The other common approach to tackle problems in unbounded domains is using infinite elements with shape functions [21-23] to map the unbounded fields into finite regions in which standard interpolation techniques can be performed [24-26].

The material properties of functionally graded materials (FGMs) can be pre-determined by controlling the spatial distribution of the composition and the variation of the volume fraction of their constituents. The FGMs had drawn attention from researchers of various disciplines in recent years. The superior performance of FGMs includes the high heat and corrosion resistances of ceramics and the high strength and toughness of metals [27-28]. In this paper, we consider elastic and crack problems made from FGMs. Cracks are commonly found in engineering structures especially during manufacturing processes involving high pressure. The analysis of isotropic FGMs of fracture mechanics under severe thermal and loading conditions had been investigated in [29-30]. Due to the near-tip stress singularity, the stress intensity factors (SIFs) are often used to evaluate the strength of cracked structures and the extension of the fatigue crack. They have the same form for homogeneous and non-homogeneous linear elastic problems [28]. Examples of evaluation of the SIFs can be found in literature [30-32]. Besides, Ozturk and Erdogan [33-34] studied the mode-I and mixed-mode cracks in an infinite non-homogeneous medium using the singular integral equation method. Kim and Paulino [35] computed the SIFs in orthotropic FGMs with the domain interaction integral method.

The finite block method (FBM) proposed recently by $\mathrm{Li}$ and Wen et al. was a strong 
form approach and had been successfully applied to deal with heat conduction [36], contact [37] and fracture mechanics [38]. The main idea of the FBM is solving PDEs in large global domain with blocks which can be mapped into normalized sub-domains. The domain discretization with blocks is more simple to implement than the mesh generation process in FEM because only a few large size blocks are required. In the mapping region, meshless approaches are employed which avoid the local mesh generation and make it easier to perform adaptive analysis. The existing strong-form FBM is not always efficient and there may not be smooth solutions to particular problems especially in the cases with complex domains or different material interfaces. Moreover, it could be complicate to incorporate boundary conditions due to the strong requirement on the continuity of approximations. In our work, we adopt in each block the meshless local Petrov-Galerkin method which is based on the weak-form formulation. Comparing with the strong-form FBM, the weak-form block Petrov-Galerkin method (BPGM) is preferred because they reduce the continuity requirements on the approximation functions and Neumann boundary conditions are satisfied naturally. The meshless basis functions are normally chosen as moving least square (MLS) approximations, RBFs or other meshless kernels. In this paper, we use the Lagrange polynomial functions whose computational effort are much less than the RBFs and MLS interpolations. Another advantage of the block method is the satisfaction of continuity at the interface of neighboring blocks. The analysis of elastic fracture mechanics in finite regions by strong form meshless finite block method had already been performed in [42]. In the present paper, the elastic and crack problems in unbounded domains are investigated also. For the sake of validating the efficiency and accuracy, numerical solutions are compared with analytical values, finite element analysis results and the solutions of meshless methods including the BEM and EFG. This paper is organized as follows: Section 2 briefly introduces formulas of the strong-form FBM for one- and two-dimensional problems. Section 3 is the mapping and coordinate transformation for finite and infinite blocks. The meshless local Petrov-Galerkin method for solid mechanics is illustrated in Section 4. Based on the previous three section, Section 5 presents the process of implementation and 
weak-form formulations of the BPGM. Five numerical examples are given in Section 6, from which all the illustrated advantages can be observed. Finally are conclusions and appendix.

\section{Strong-form Finite block method}

The strong form FBM was proposed and developed by Wen et al. in recent years and had been numerically proved with high accuracy and efficiency. The main idea of this approach is to discretize the problem domain into several sub-regions, named blocks, which can be mapped into normalized domains in which point collocation schemes can be employed. Once the real domain is transformed to normalized domain by using quadratic type of blocks, the partial differential operators in practical region will be computed by differential operators in the normalized space. This section is a brief introduction of the strong form FBM for one- and two-dimensional problems using Lagrange series of interpolation. More details can be referred to [36-40].

\subsection{One dimensional case}

Consider a set of uniformly distributed nodes in normalized domain as shown in Figure $1, \quad \xi_{i}=-1+2(i-1) /(n-1), i=1,2, \ldots, n .[34]$

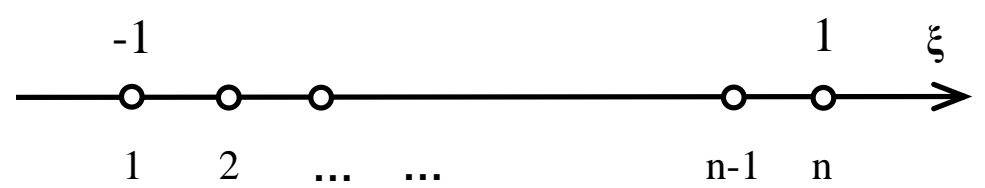

Figure 1. One-dimensional node distribution in normalized domain.

The function $u(\xi)$ can be evaluated by the use of Lagrange polynomials as

$$
u(\xi)=\sum_{i=1}^{n} L\left(\xi, \xi_{i}\right) u_{i}
$$

where

$$
L\left(\xi, \xi_{i}\right)=\prod_{\substack{k=1 \\ k \neq i}}^{n} \frac{\left(\xi-\xi_{k}\right)}{\left(\xi_{i}-\xi_{k}\right)} .
$$


Then the first order derivative can be determined by

$$
\frac{\partial u(\xi)}{\partial \xi}=\sum_{i=1}^{n} \frac{\partial L\left(\xi, \xi_{i}\right)}{\partial \xi} u_{i}
$$

where

$$
\frac{\partial L\left(\xi, \xi_{i}\right)}{\partial \xi}=\sum_{j=1}^{n} \frac{\prod_{k=1, k \neq i, k \neq j}^{n}\left(\xi-\xi_{k}\right)}{\prod_{k=1, k \neq i}^{n}\left(\xi_{i}-\xi_{k}\right)}
$$

which can be briefly written in matrix form as

$$
\mathbf{u}_{\xi}=\mathbf{D u},
$$

in which $\mathbf{u}_{\xi}$ is the vector of nodal value of first order derivative with respect to $\mathbf{u}$,

D is the first order differential matrix whose entries can be calculated by Eq.(4).

\subsection{Two-dimensional case}

For a set of nodes $\left(\xi_{k_{1}}, \eta_{k_{2}}\right), k_{1}=1,2, \ldots, n_{1}, k_{2}=1,2, \ldots, n_{2}$, in the normalized domain with $\left|\xi_{k_{1}}\right| \leq 1,\left|\eta_{k_{2}}\right| \leq 1$, The function $u(\xi, \eta)$ can be approximated by using Lagrange polynomials as [42]:

$$
u(\xi, \eta)=\sum_{i=1}^{n_{1}} \sum_{j=1}^{n_{2}} F\left(\xi, \xi_{i}\right) G\left(\eta, \eta_{j}\right) u_{i j}
$$

where

$$
F\left(\xi, \xi_{i}\right)=\prod_{l=1, l \neq i}^{n_{1}}\left(\frac{\xi-\xi_{l}}{\xi_{i}-\xi_{l}}\right), \quad G\left(\eta, \eta_{j}\right)=\prod_{m=1, m \neq j}^{n_{2}}\left(\frac{\eta-\eta_{m}}{\eta_{j}-\eta_{m}}\right)
$$

the subscript $i j=n_{1}(j-1)+i$ and $\left\{u_{i j}\right\}_{i j=1}^{N}$ are nodal values as shown in Figure 2. 


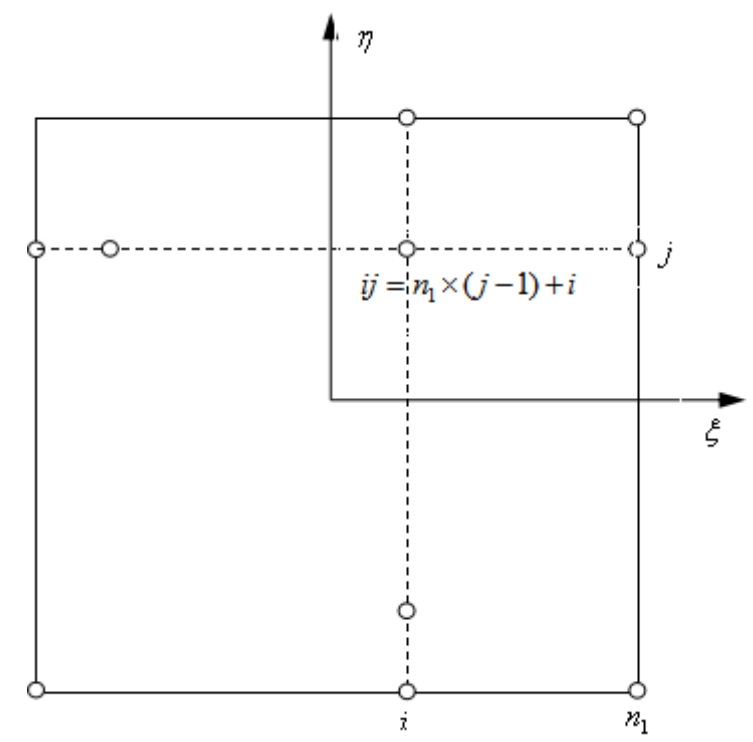

Figure 2: The number system of nodes in normalized domain.

The first order derivative of $u$ with respect to $\xi$ can be represented as:

$$
\frac{\partial u(\xi, \eta)}{\partial \xi}=\sum_{i=1}^{n_{1}} \sum_{j=1}^{n_{2}} \frac{\partial F\left(\xi, \xi_{i}\right)}{\partial \xi} G\left(\eta, \eta_{j}\right) u_{i j},
$$

where

$$
\frac{\partial F\left(\xi, \xi_{i}\right)}{\partial \xi}=\frac{\partial}{\partial \xi}\left[\prod_{l=1, l \neq i}^{n_{1}}\left(\frac{\xi-\xi_{l}}{\xi_{i}-\xi_{l}}\right)\right]=\sum_{p=1}^{n_{1}}\left[\frac{\prod_{q=1, q \neq i, q \neq p}^{n_{1}}\left(\xi-\xi_{q}\right)}{\prod_{l=1, l \neq i}^{n_{1}}\left(\xi_{i}-\xi_{l}\right)}\right] .
$$

Similarly, the first order derivative with respect to $\eta$ can be represented as

$$
\frac{\partial u(\xi, \eta)}{\partial \eta}=\sum_{i=1}^{n_{1}} \sum_{j=1}^{n_{2}} F\left(\xi, \xi_{i}\right) \frac{\partial G\left(\eta, \eta_{j}\right)}{\partial \eta} u_{i j}
$$

where

$$
\frac{\partial G\left(\eta, \eta_{j}\right)}{\partial \eta}=\frac{\partial}{\partial \eta}\left[\prod_{m=1, m \neq j}^{n_{2}} \frac{\left(\eta-\eta_{m}\right)}{\left(\eta_{j}-\eta_{m}\right)}\right]=\sum_{p=1}^{n_{2}}\left[\frac{\prod_{q=1, q \neq j, q \neq p}^{n_{2}}\left(\eta-\eta_{q}\right)}{\prod_{m=1, m \neq j}^{n_{2}}\left(\eta_{j}-\eta_{m}\right)}\right] .
$$

For the sake of simplicity, we briefly write Eq.(6), (8) and (10) as

$$
u=\sum_{i=1}^{n_{1}} \sum_{j=1}^{n_{2}} F_{i} G_{j} u_{i j}, \quad u_{\xi}=\sum_{i=1}^{n_{1}} \sum_{j=1}^{n_{2}} F_{i, \xi} G_{j} u_{i j}, \quad u_{\eta}=\sum_{i=1}^{n_{1}} \sum_{j=1}^{n_{2}} F_{i} G_{j, \eta} u_{i j},
$$

where $F_{i}=F\left(\xi, \xi_{i}\right), G_{j}=G\left(\eta, \eta_{j}\right), F_{i, \xi}=\partial F_{i} / \partial \xi, G_{j, \eta}=\partial G_{j} / \partial \eta$. Extending this idea to high order derivatives, for example, the $n^{\xi-\text { th }}$ and $n^{\eta \text { th }}$ derivatives with 
respective to $\xi$ and $\eta$, we have:

$$
u_{\xi^{\eta^{\xi}} n^{\eta}}(\xi, \eta)=\frac{\partial u^{n^{\xi}+n^{\eta}}(\xi, \eta)}{\partial \xi^{n^{\xi}} \partial \eta^{n^{\eta}}}=\sum_{i=1}^{n_{1}} \sum_{j=1}^{n_{2}}\left(F_{i, \xi}\right)^{n^{\xi}}\left(G_{j, \eta}\right)^{n^{\eta}} u_{i j}
$$

It means that high order derivatives can be obtained directly by the first order derivatives. Therefore, the computational recursion is avoided.

We can also write the derivatives in matrix form based on one-dimensional first order derivative. The partial differentials at each node with respect to $\xi$ can be written as

$$
\mathbf{u}_{\xi}=\mathbf{D}_{\xi} \mathbf{u}
$$

where $\mathbf{u}_{\xi}=\left[u_{\xi_{1}}, \ldots, u_{\xi_{m}}\right]$ is the vector of derivative nodal values, $m\left(=n_{1} \times n_{2}\right)$ is the total number of collocation points (as shown in Figure 2) and

$$
\mathbf{D}_{\xi}=\underbrace{\left[\begin{array}{cccc}
\mathbf{D} & 0 & \cdots & 0 \\
0 & \mathbf{D} & \cdots & 0 \\
\cdots & \cdots & \cdots & \cdots \\
0 & 0 & \cdots & \mathbf{D}
\end{array}\right]}_{n_{2}}
$$

in which $\mathbf{D}$ is the one-dimensional differential matrix as given in Eq.(5) with dimension $n_{1} \times n_{1}$. The partial differentials with respect to $\eta$ should be constructed in the numbering system with the number of nodes $p=n_{2} \times(i-1)+j$, which can be written as:

$$
\mathbf{u}_{\eta}=\mathbf{D}_{\eta} \mathbf{u}
$$

By using the transform matrix $\mathbf{T}$ in which all elements are zero except $\mathbf{T}_{n_{1} \times(j-1)+i, n_{2} \times(i-1)+j}=1,\left(i=1,2, \ldots, n_{1}, j=1,2, \ldots, n_{2}\right)$, we have:

$$
\mathbf{D}_{\eta}=\mathbf{T D}_{\xi} \mathbf{T}^{-1}, \quad \mathbf{T}^{-1}=\mathbf{T}^{T}
$$

Therefore, high order derivatives can be denoted in matrix form as

$$
\mathbf{u}_{\xi \eta}^{(r, s)}=\mathbf{D}_{\xi}^{r} \mathbf{D}_{\eta}^{s} \mathbf{u}
$$

This shows again that high order and multi-dimensional partial differentials at all the nodal points can be calculated by one-dimensional differential matrix of first order. 


\section{Mapping and coordinate transformation}

\subsection{Finite blocks}

For two-dimensional problems in finite regions, quadratics type of block with 8 seeds as shown in Figure 3 is often employed to map physical domains into normalized domains. The shape functions at each seed are:

$$
\begin{aligned}
& N_{i}=\frac{1}{4}\left(1+\xi_{i} \xi\right)\left(1+\eta_{i} \eta\right)\left(\xi_{i} \xi+\eta_{i} \eta-1\right) \quad(i=1,2,3,4), \\
& N_{i}=\frac{1}{2}\left(1-\xi^{2}\right)\left(1+\eta_{i} \eta\right) \quad(i=5,7), \\
& N_{i}=\frac{1}{2}\left(1-\eta^{2}\right)\left(1+\xi_{i} \xi\right) \quad(i=6,8),
\end{aligned}
$$

which can be found in previous works of strong form FBM [36-40]. The corresponding partial differentials are given in appendix.
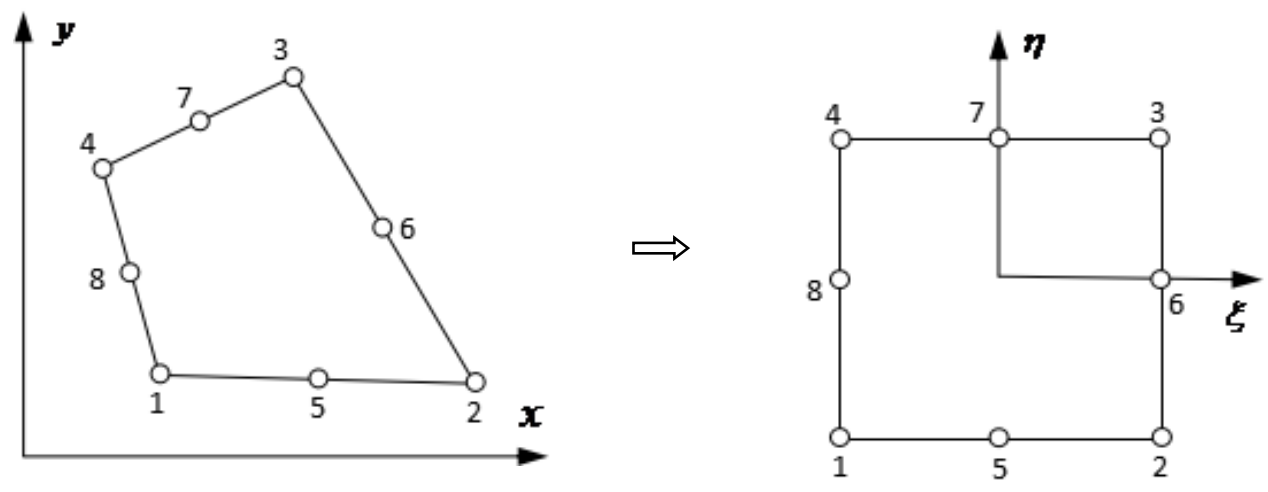

Figure 3: Mapping of finite block: physical domain (left) and normalized domain (right).

\subsection{Infinite blocks}

To solve problems in unbounded regions, infinite domain blocks are set up on definite places which are chosen artificially to model infinite boundaries. The shape functions in these blocks can be created with different number of seeds, among which the widely used are: four-seed mapping, five-seed mapping and six-seed mapping [24-26] as shown in Figure 4. 


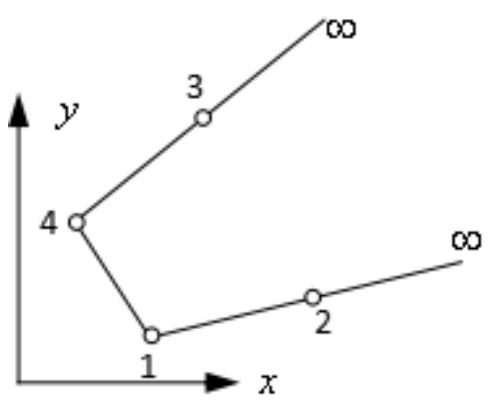

(a)

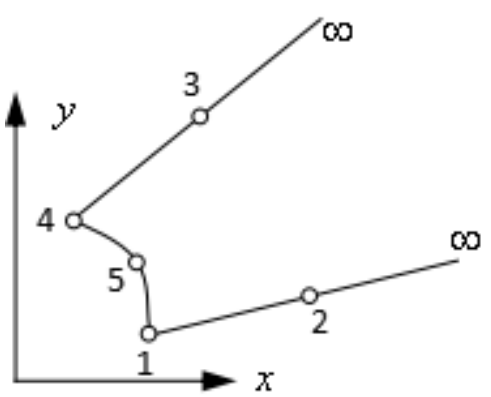

(c)

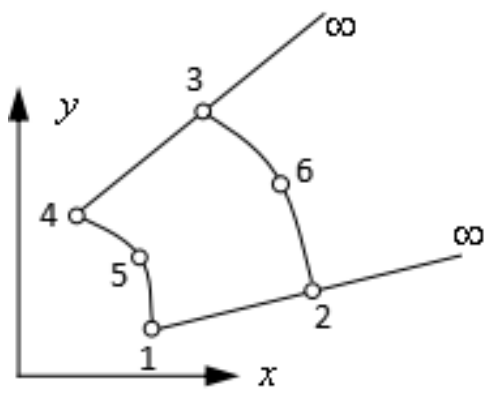

(e)

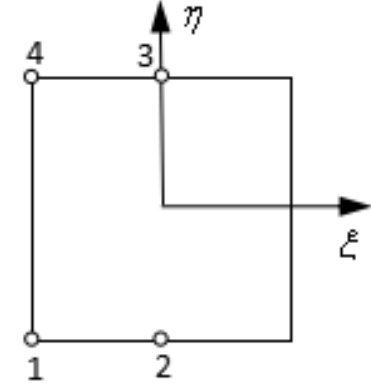

(b)

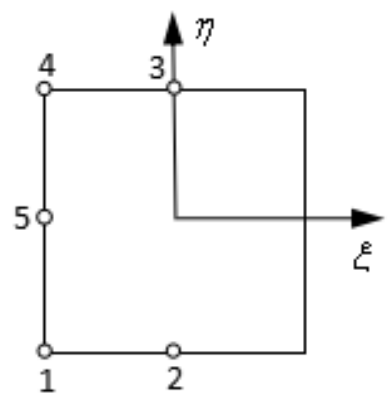

(d)

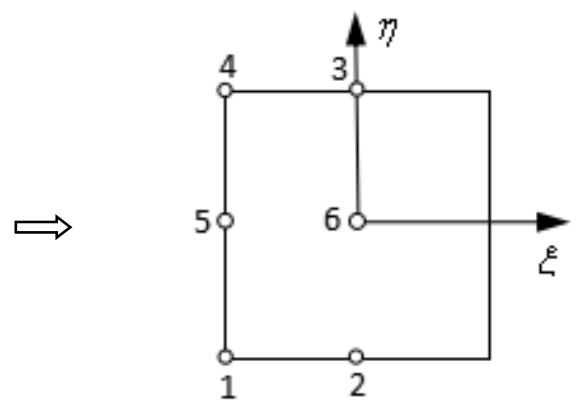

(f)

Figure 4: Mapping for infinite blocks between physical (left) and normalized (right) domains with: four seeds $(a, b)$, five seeds $(c, d)$ and six seeds $(e, f)$.

The corresponding shape functions in infinite blocks are:

four-seed mapping:

$$
N_{1}=-\frac{\xi(1-\eta)}{(1-\xi)}, N_{2}=\frac{(1+\xi)(1-\eta)}{2(1-\xi)}, N_{3}=\frac{(1+\xi)(1+\eta)}{2(1-\xi)}, N_{4}=-\frac{\xi(1+\eta)}{(1-\xi)},
$$

five-seed mapping: 


$$
\begin{aligned}
& N_{1}=-\frac{(1+\xi+\eta)(1-\eta)}{(1-\xi)}, N_{2}=\frac{(1+\xi)(1-\eta)}{2(1-\xi)}, N_{3}=\frac{(1+\xi)(1+\eta)}{2(1-\xi)}, \\
& N_{4}=\frac{(-1+\eta-\xi)(1+\eta)}{(1-\xi)}, N_{5}=\frac{2\left(1-\eta^{2}\right)}{(1-\xi)}
\end{aligned}
$$

six-seed mapping:

$$
\begin{aligned}
& N_{1}=-\frac{\xi\left(\eta^{2}-\eta\right)}{(1-\xi)}, N_{2}=\frac{(1+\xi)\left(\eta^{2}-\eta\right)}{2(1-\xi)}, N_{3}=\frac{(1+\xi)\left(\eta^{2}+\eta\right)}{2(1-\xi)}, \\
& N_{4}=-\frac{\xi\left(\eta^{2}+\eta\right)}{(1-\xi)}, N_{5}=-\frac{2 \xi\left(1-\eta^{2}\right)}{(1-\xi)}, N_{6}=\frac{(1+\xi)\left(1-\eta^{2}\right)}{(1-\xi)}
\end{aligned}
$$

whose partial differentials with respect to normalized axes $\xi$ and $\eta$ are listed in appendix.

By the use of shape functions, the coordinate transformation can be determined as:

$$
x=\sum_{k=1}^{n} N_{k}(\xi, \eta) x_{k}, \quad y=\sum_{k=1}^{n} N_{k}(\xi, \eta) y_{k},
$$

where $n$ is the number of seeds, $\left(x_{k}, y_{k}\right)$ and $N_{k}(\xi, \eta)$ are coordinate and shape functions at the $k^{\text {th }}$ seed in the real domain.

Therefore, partial differentials of function $u(x, y)$ can be expressed as:

$$
\frac{\partial u}{\partial x}=\frac{1}{J}\left(\beta_{11} \frac{\partial u}{\partial \xi}+\beta_{12} \frac{\partial u}{\partial \eta}\right), \frac{\partial u}{\partial y}=\frac{1}{J}\left(\beta_{21} \frac{\partial u}{\partial \xi}+\beta_{22} \frac{\partial u}{\partial \eta}\right)
$$

in which $J=\left|\begin{array}{ll}\frac{\partial x}{\partial \xi} & \frac{\partial x}{\partial \eta} \\ \frac{\partial y}{\partial \xi} & \frac{\partial y}{\partial \eta}\end{array}\right|=\beta_{22} \beta_{11}-\beta_{21} \beta_{12}, \quad \beta_{11}=\frac{\partial y}{\partial \eta}, \beta_{12}=-\frac{\partial y}{\partial \xi}, \beta_{21}=-\frac{\partial x}{\partial \eta}, \beta_{22}=\frac{\partial x}{\partial \xi}$.

Substituting Eq.(8) and (10) into Eq.(24), we have:

$$
\begin{aligned}
& \frac{\partial u}{\partial x}=\frac{1}{J} \sum_{i=1}^{n_{1}} \sum_{j=1}^{n_{2}}\left(\beta_{11} F_{i, \xi} G_{j}+\beta_{12} F_{i} G_{j, \eta}\right) u_{i j}, \\
& \frac{\partial u}{\partial y}=\frac{1}{J} \sum_{i=1}^{n_{1}} \sum_{j=1}^{n_{2}}\left(\beta_{21} F_{i, \xi} G_{j}+\beta_{22} F_{i} G_{j, \eta}\right) u_{i j} .
\end{aligned}
$$

Note that shape functions at seeds of quadratic finite and infinite blocks are used for mapping of geometries while the Lagrange interpolation polynomial are employed for approximation of differential operators. In addition, the degree of freedom for each block could be different between each block. 


\section{Meshless local Petrov-Galerkin method for solid mechanics}

For a two-dimensional linear, elastic boundary value problem in a global domain $\Omega$ bounded by $\Gamma$, the force equilibrium equations can be written as:

$$
\begin{aligned}
& \frac{\partial \sigma_{x}}{\partial x}+\frac{\partial \tau_{x y}}{\partial y}=f_{x}, \\
& \frac{\partial \tau_{x y}}{\partial x}+\frac{\partial \sigma_{y}}{\partial y}=f_{y},(x, y) \in \Omega
\end{aligned}
$$

with boundary conditions:

$$
\begin{gathered}
u(x, y)=\bar{u}(x, y), v(x, y)=\bar{v}(x, y),(x, y) \in \Gamma_{u} \\
t_{x}(x, y)=\bar{t}_{x}(x, y), t_{y}(x, y)=\bar{t}_{y}(x, y),(x, y) \in \Gamma_{q}
\end{gathered}
$$

where $f_{x}, f_{y}$ denote body forces, $\sigma_{x}, \sigma_{y}$ are normal stresses with respect to coordinate direction $x$ and $y, \tau_{x y}$ is shear stress. $u, v$ denote displacements, $t_{x}$ and $t_{y}$ are tractions along the direction of outer normal vectors $\left(n_{x}, n_{y}\right)$, that is:

$$
\begin{aligned}
& t_{x}=\sigma_{x} n_{x}+\tau_{x y} n_{y}, \\
& t_{y}=\tau_{x y} n_{x}+\sigma_{y} n_{y} .
\end{aligned}
$$

Using meshless local Petrov-Galerkin method, the weak form of governing differential equations over a local integral sub-domain $\Omega_{s}$ centered at point $\mathrm{x}_{s}\left(x_{s}, y_{s}\right)$ can be obtained [10] (Figure 5).
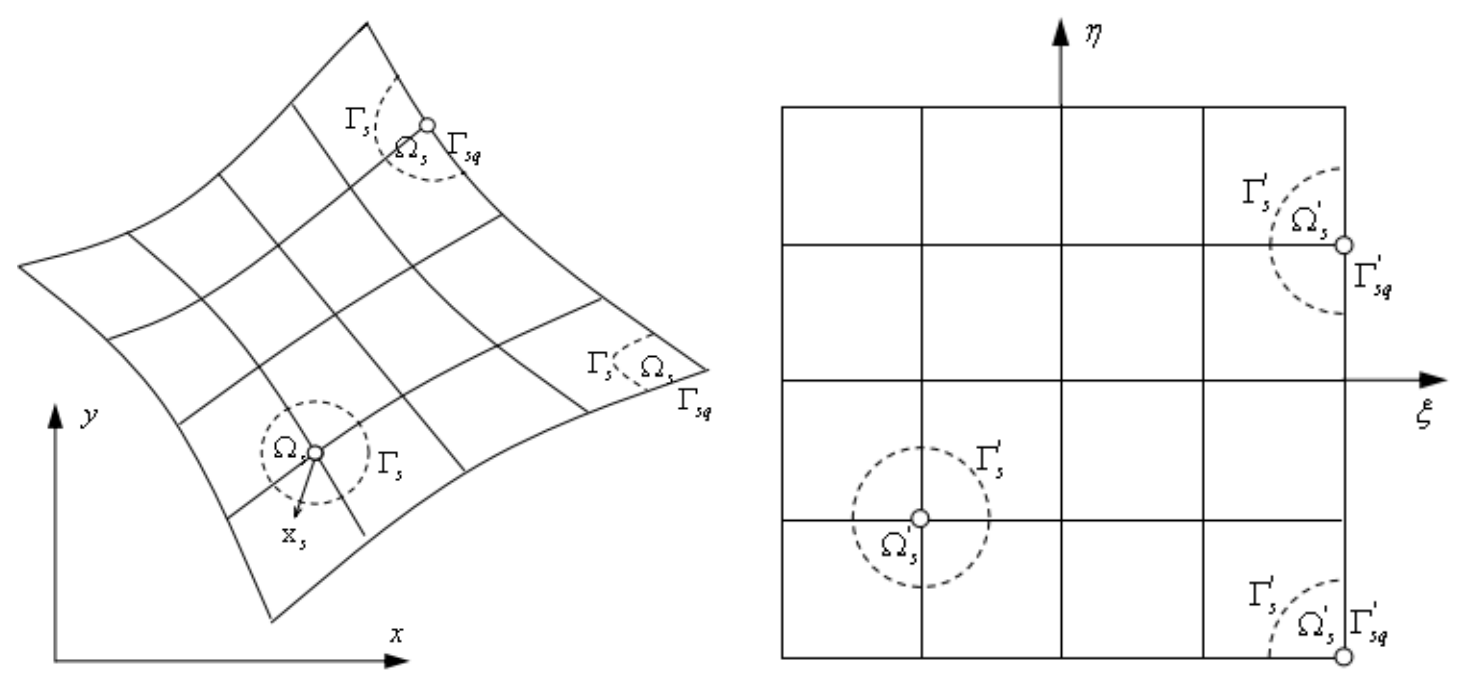
Figure 5: Local integral domain in the physical (left) and normalized domain (right).

The weak formulation for the first equation in Eq.(26) is given as:

$$
\int_{\Omega_{s}}\left(\frac{\partial \sigma_{x}}{\partial x}+\frac{\partial \tau_{x y}}{\partial y}-f_{x}\right) \phi^{*} d \Omega=0
$$

where $\phi^{*}$ is a test function. By the use of the divergence theorem, the weak form can be expressed as:

$$
\int_{\Gamma_{s}}\left(\sigma_{x} n_{x}+\tau_{x y} n_{y}\right) \phi^{*} d \Gamma-\int_{\Omega_{s}}\left(\sigma_{x} \frac{\partial \phi^{*}}{\partial x}+\tau_{x y} \frac{\partial \phi^{*}}{\partial y}+f_{x} \phi^{*}\right) d \Omega=0
$$

where $\Gamma_{s}$ is the boundary of the local integral domain $\Omega_{s}$. In this paper, we choose the simplest test function as follows:

$$
\phi^{*}(x, y)=\left\{\begin{array}{ll}
1, & (x, y) \in \Omega_{s} \\
0, & (x, y) \notin \Omega_{s}
\end{array} .\right.
$$
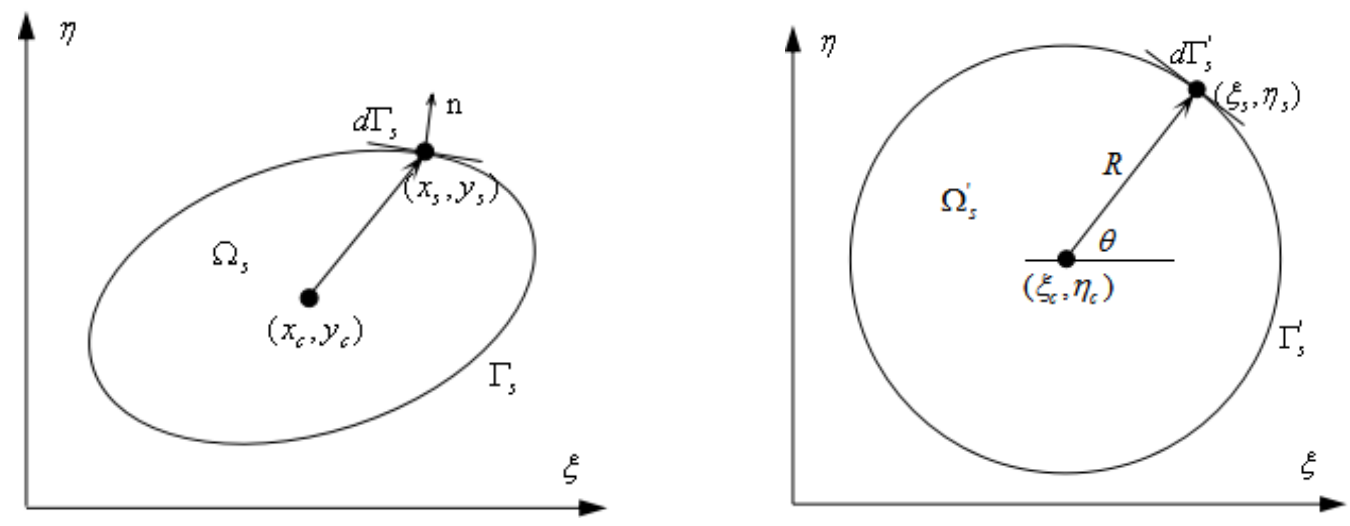

Figure 6: Local integral mapping: physical (left) and normalized (right) domain.

To consider the local integral mapping in $\Omega_{s}$ as shown in Figure 6, the normalized local integral region for point $\left(x_{c}, y_{c}\right)$ is selected as a circle of radius $R$ and centered at $\left(\xi_{c}, \eta_{c}\right)$. Then the coordinate of points at the local integral boundary can be written as:

$$
\xi=\xi_{c}+R \cos \theta, \quad \eta=\eta_{c}+R \sin \theta .
$$

Coupled with coordinate transform formula Eq.(23), the components of the line 
segment in physical domain are:

$$
\begin{aligned}
& d x=\left[\sum_{k=1}^{n}\left(-\frac{\partial N_{k}(\xi, \eta)}{\partial \xi} \sin \theta+\frac{\partial N_{k}(\xi, \eta)}{\partial \eta} \cos \theta\right) x_{k}\right] R d \theta=g(\xi, \eta) R d \theta, \\
& d y=\left[\sum_{k=1}^{n}\left(-\frac{\partial N_{k}(\xi, \eta)}{\partial \xi} \sin \theta+\frac{\partial N_{k}(\xi, \eta)}{\partial \eta} \cos \theta\right) y_{k}\right] R d \theta=h(\xi, \eta) R d \theta .
\end{aligned}
$$

Therefore, the length of the local integral boundary segment is determined as:

$$
d \Gamma=\sqrt{d x^{2}+d y^{2}}=\sqrt{g^{2}+h^{2}} R d \theta .
$$

The components of the outer normal vectors are:

$$
n_{x}=\cos (\alpha-\pi / 2), \quad n_{y}=\sin (\alpha-\pi / 2)
$$

where $\alpha$ is the tangential at this point in the Cartesian coordinate:

$$
\alpha=\tan ^{-1} \frac{d y}{d x} \text {. }
$$

\section{Weak-form block Petrov-Galerkin method in functionally graded materials}

\subsection{The process of implementation}

The execution procedure of BPGM is briefly as follows:

Step 1. Discretize the problem domain into a set of blocks with four sides: straight or curved, and remember the connectivity of neighboring blocks.

Step 2. Map each block from physical region to normalized square domain and determine the coordinate transformation by the use of shape functions.

Step 3. Construct equations in each sub-region based on the weak-form Petrov-Galerkin technique with Lagrange interpolation calculation.

Step 4. Assemble equations in all the sub-regions as well as those for continuity conditions at the interface of neighboring blocks into the system equation.

Step 5. Exert boundary conditions.

Step 6. Solve system equations and evaluate values at place of interest.

\subsection{Weak-form equations in FGMs}

The material properties in non-homogeneous functionally graded materials are dependent on the spatial coordinates. For plane stress elasticity, the relationship 
between stresses and strains gives:

$$
\begin{aligned}
& \sigma_{x}=Q_{11} \frac{\partial u}{\partial x}+Q_{12} \frac{\partial v}{\partial y} \\
& \sigma_{y}=Q_{21} \frac{\partial u}{\partial x}+Q_{22} \frac{\partial v}{\partial y}, \\
& \tau_{x y}=Q_{3}\left(\frac{\partial u}{\partial y}+\frac{\partial v}{\partial x}\right)
\end{aligned}
$$

in which $Q_{11}, Q_{12}, Q_{21}, Q_{22}$ and $G$ are material coefficients as follows:

$$
Q_{11}=\frac{E_{1}}{1-v^{2}}, Q_{12}=Q_{21}=\frac{v E_{1}}{1-v^{2}}, Q_{22}=\frac{E_{2}}{1-v^{2}}, Q_{3}=G
$$

where $E_{1}$ and $E_{2}$ are Young's modulus along two axis of coordinate, $v$ is Possion's ratio and $G$ is the shear modulus.

By substituting Eq.(25) into Eq.(38), then into Eq. (31), we have:

$$
\begin{aligned}
\int_{\Gamma_{s}} & \frac{1}{J} \sum_{i=1}^{n_{1}} \sum_{j=1}^{n_{2}}\left\{\left[Q_{11}\left(\beta_{11} F_{i, \xi} G_{j}+\beta_{12} F_{i} G_{j, \eta}\right) u_{i j}+Q_{12}\left(\beta_{12} F_{i, \xi} G_{j}+\beta_{22} F_{i} G_{j, \eta}\right) v_{i j}\right] n_{x}\right. \\
& \left.+Q_{3}\left[\left(\beta_{12} F_{i, \xi} G_{j}+\beta_{22} F_{i} G_{j, \eta}\right) u_{i j}+\left(\beta_{11} F_{i, \xi} G_{j}+\beta_{12} F_{i} G_{j, \eta}\right) v_{i j}\right] n_{y}\right\} d \Gamma=\int_{\Omega_{s}} f_{x} d \Omega
\end{aligned}
$$

The Dirichlet boundary conditions can be satisfied directly by Eq.(27). When Neumann conditions are given at the boundary, we have the following weak formulation:

$$
\begin{aligned}
& \int_{\Gamma_{s}-\Gamma_{s q}} \frac{1}{J} \sum_{i=1}^{n_{1}} \sum_{j=1}^{n_{2}}\left\{\left[Q_{11}\left(\beta_{11} F_{i, \xi} G_{j}+\beta_{12} F_{i} G_{j, \eta}\right) u_{i j}+Q_{12}\left(\beta_{12} F_{i, \xi} G_{j}+\beta_{22} F_{i} G_{j, \eta}\right) v_{i j}\right] n_{x}\right. \\
& \left.\quad+Q_{3}\left[\left(\beta_{12} F_{i, \xi} G_{j}+\beta_{22} F_{i} G_{j, \eta}\right) u_{i j}+\left(\beta_{11} F_{i, \xi} G_{j}+\beta_{12} F_{i} G_{j, \eta}\right) v_{i j}\right] n_{y}\right\} d \Gamma+\int_{\Gamma_{s q}} \bar{t}_{x} d \Gamma \\
& =\int_{\Omega_{s}} f_{x} d \Omega .
\end{aligned}
$$

Rewrite the length of boundary segment by using Eq.(35), we have:

$$
\begin{aligned}
& \int_{\Gamma_{s}} \frac{R}{J} \sum_{i=1}^{n_{1}} \sum_{j=1}^{n_{2}}\left\{\left[Q_{11}\left(\beta_{11} F_{i, \xi} G_{j}+\beta_{12} F_{i} G_{j, \eta}\right) u_{i j}+Q_{12}\left(\beta_{12} F_{i, \xi} G_{j}+\beta_{22} F_{i} G_{j, \eta}\right) v_{i j}\right] n_{x}\right. \\
& \left.\quad+Q_{3}\left[\left(\beta_{12} F_{i, \xi} G_{j}+\beta_{22} F_{i} G_{j, \eta}\right) u_{i j}+\left(\beta_{11} F_{i, \xi} G_{j}+\beta_{12} F_{i} G_{j, \eta}\right) v_{i j}\right] n_{y}\right\} u_{i j} \sqrt{g^{2}+h^{2}} d \theta \\
& =f_{x} A\left(\mathbf{x}_{s}\right)
\end{aligned}
$$

for Eq.(40) and 


$$
\begin{aligned}
& \int_{\Gamma_{s}-\Gamma_{s q}} \frac{R}{J} \sum_{i=1}^{n_{1}} \sum_{j=1}^{n_{2}}\left\{\left[Q_{11}\left(\beta_{11} F_{i, \xi} G_{j}+\beta_{12} F_{i} G_{j, \eta}\right) u_{i j}+Q_{12}\left(\beta_{12} F_{i, \xi} G_{j}+\beta_{22} F_{i} G_{j, \eta}\right) v_{i j}\right] n_{x}\right. \\
& \left.\quad+Q_{3}\left[\left(\beta_{12} F_{i, \xi} G_{j}+\beta_{22} F_{i} G_{j, \eta}\right) u_{i j}+\left(\beta_{11} F_{i, \xi} G_{j}+\beta_{12} F_{i} G_{j, \eta}\right) v_{i j}\right] n_{y}\right\} u_{i j} \sqrt{g^{2}+h^{2}} d \theta \\
& +\int_{\Gamma_{s q}} \bar{t}_{x} \sqrt{g^{2}+h^{2}} R d \theta=f_{x} A\left(\mathbf{x}_{s}\right) .
\end{aligned}
$$

for Eq.(41), where $A\left(\mathrm{x}_{s}\right)$ is the area of the local integral domain $\Omega_{s}$.

\section{Numerical results}

\section{Example 6.1: 2D beam}

Consider a 2D beam with height $h$ unit and length $L$ unit in orthotropic functionally graded media with tension $P$ unit as shown in Figure 7 . In the computation, the geometric parameters and the mechanical properties of materials are normalized as: $L=5 h, P=1, Q_{11}=e^{y / h}, Q_{22}=e^{x / L}, Q_{12}=1 / 3$ and $Q_{3}=1 / 2$. Based on the classical Euler-Bernoulli beam theory, the analytical expression of normal stress $\sigma$, shear stress $\tau$ and vertical deflection of top surface $u_{y}(x, h)$ are given as [40]:

$$
\begin{aligned}
& \sigma=-\frac{e-1}{e^{2}-3 e+1} \frac{P(L-x)}{h^{3}}(y-a) e^{y / h}, \\
& \tau=\frac{(e-1) P}{\left(e^{2}-3 e+1\right) h^{2}}\left[(h-y+a) e^{y / h}-a e\right], \\
& u_{y}(x, h)=\frac{P(e-1)}{6 Q_{0} h^{3}\left(e^{2}-3 e+1\right)} x^{2}(3 L-x),
\end{aligned}
$$

where $a=h /(e-1)$.

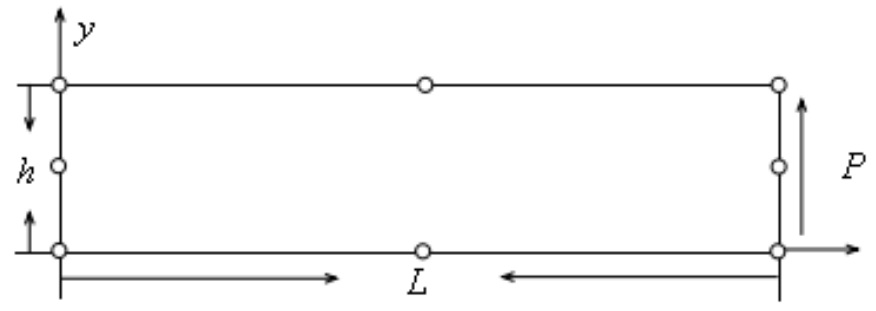

Figure 7: Cantilever beam with 8 mapping seeds ( $\mathrm{o}$ ).

For this problem, we adopt one finite block with 8 seeds (Figure 7) and a set of 
$N^{2}$ uniformly distributed nodes in this block. Numerical results of normal and shear stresses at the cross section $x=L / 2$ are given in Figure 8 and 9 , respectively. Figure 10 shows that the deflection of this beam at the top surface with respect to different number of nodes. It is evident that all the numerical solutions are in good agreement with analytical values. When the number of nodes in the block equals to 13 at each dimension, Table 1 presents relative errors of the point with the largest shear stress at the middle section using various different local integral radius $R$, which is chosen to be $k \cdot \Delta$, where $\Delta$ is one fourth of the distance between two neighboring nodes in the mapping domain (Figure 2: right) and $k$ is a given constant. We can observe that the radius $R$ for local integral has little impact on the degree of accuracy when a relatively small integral domain is selected.

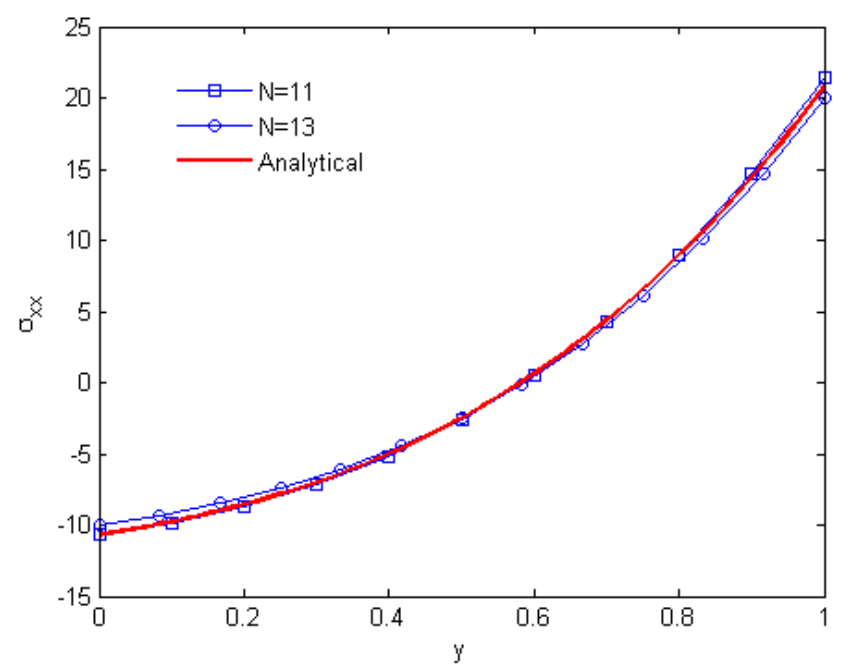

Figure 8: Numerical and analytical normal stress distribution at middle section with $x=L / 2$. 


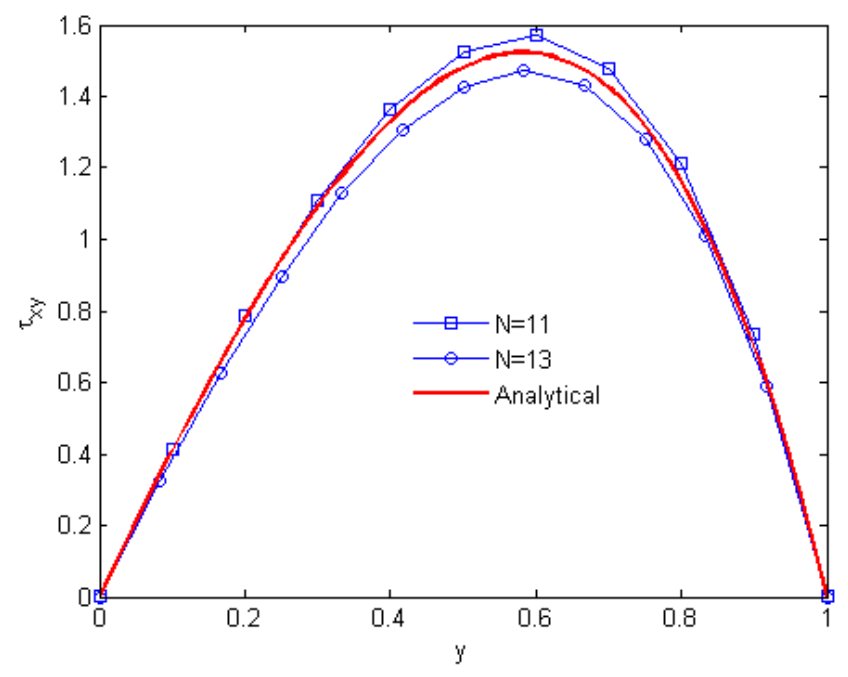

Figure 9: Numerical and analytical shear stress distribution at middle section with $x=L / 2$.

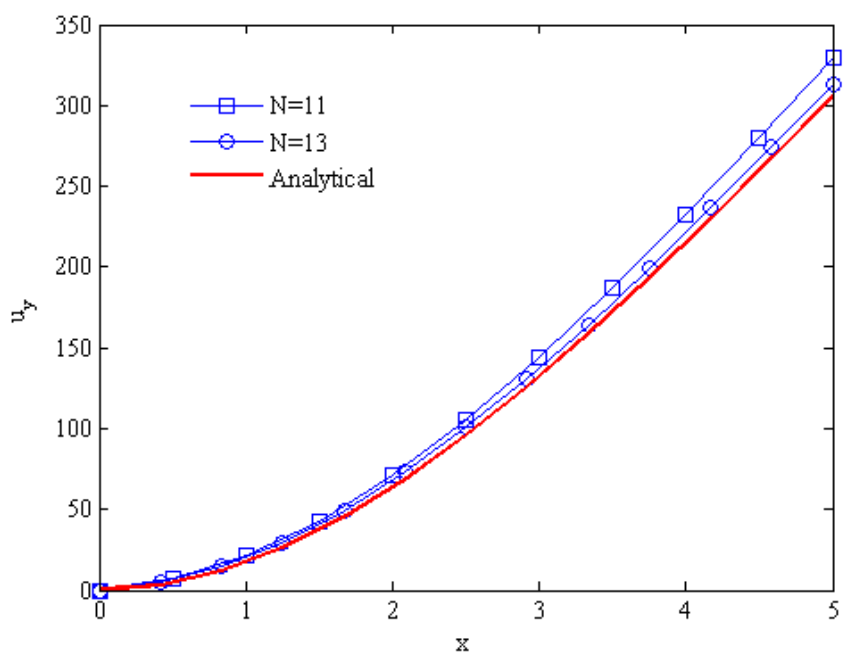

Figure 10: Numerical and analytical vertical deflection at the top surface.

Table 1: Relative error of the point contributes to the largest $\tau_{x y}$ in Figure 8 using various different local integral radius.

\begin{tabular}{c|cccccccccc}
\hline$k$ & 0.9 & 0.8 & 0.7 & 0.6 & 0.5 & 0.4 & 0.3 & 0.2 & 0.1 & 0.05 \\
\hline$R\left(\times 10^{-2}\right)$ & 3.75 & 3.33 & 2.92 & 2.50 & 2.08 & 1.67 & 1.25 & 0.83 & 0.42 & 0.21 \\
\hline Error $(\%)$ & 7.27 & 3.91 & 1.07 & 1.28 & 3.22 & 4.12 & 5.84 & 6.69 & 7.10 & 6.81 \\
\hline
\end{tabular}

\section{Example 6.2: Rectangular plate containing a central crack}

Consider a rectangular plate of width $2 w$ unit and height $2 h$ unit with a central crack of length $2 a$ unit under uniformly distributed load $\sigma_{0}$ on two ends as shown in Figure 11 (left). Due to the symmetry of this problem, only a quarter plate is 
analyzed with dimension $h$ equals to $a$.
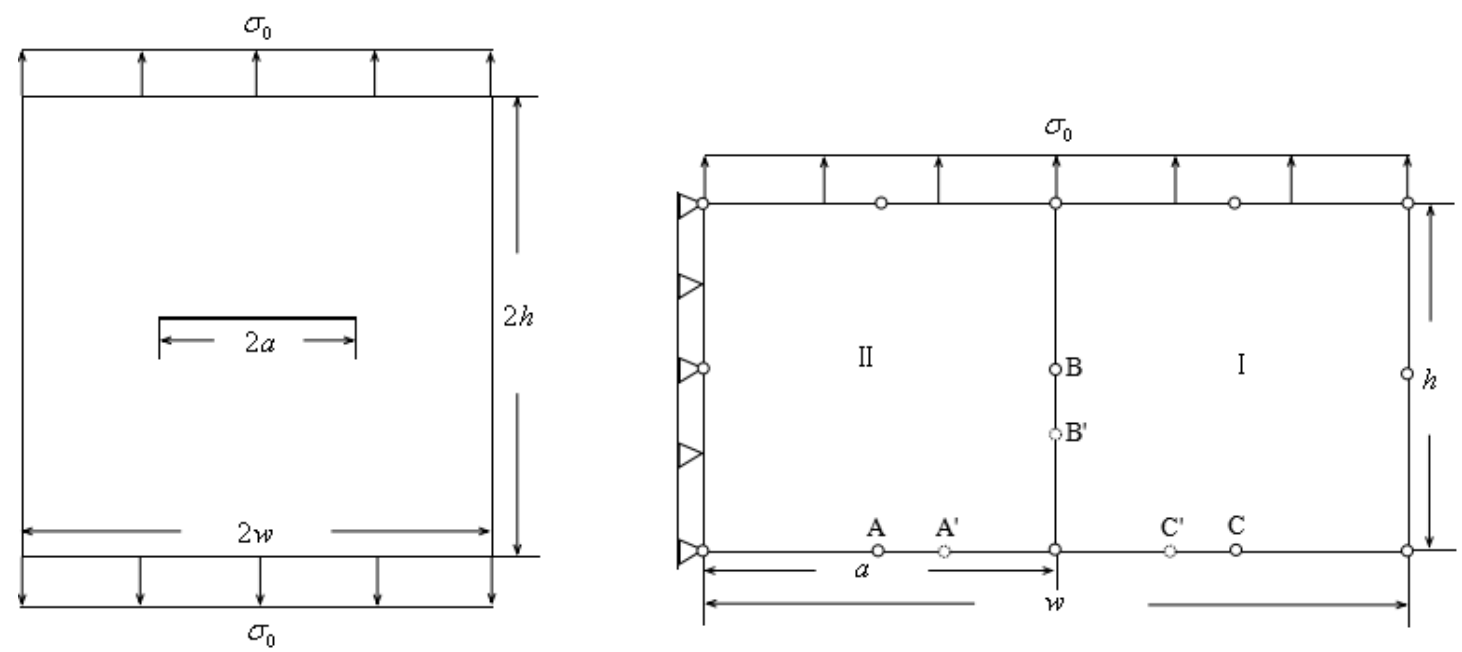

Figure 11: Center-cracked plate with tensile loads (left) and an enlarged quarter of this (right).

For this problem, two finite blocks introduced in section 3.1 are employed. The displacements and stresses for nodes at the interface $\left(\Gamma_{\text {int }}\right)$ of block I and block II except two ends (joints) are required to be compatible, that is:

$$
\begin{array}{ccc}
u_{x}^{I}(x, y)-u_{x}^{I I}(x, y)=0, & u_{y}^{I}(x, y)-u_{y}^{I I}(x, y)=0, \quad(x, y) \in \Gamma_{\mathrm{int}} \\
t_{x}^{I}(x, y)+t_{x}^{I I}(x, y)=0, & t_{y}^{I}(x, y)+t_{y}^{I I}(x, y)=0 .
\end{array}
$$

At corner joint (Figure 12), the following displacement continuity conditions and point equilibrium equations should be satisfied:

$$
\begin{gathered}
u_{x}^{I}(\mathrm{x})=u_{x}^{I I}(\mathrm{x})=\ldots=u_{x}^{K}(\mathrm{x}), \\
u_{y}^{I}(\mathrm{x})=u_{y}^{I I}(\mathrm{x})=\ldots=u_{y}^{K}(\mathrm{x}), \\
\sum_{p=I}^{K}\left(\sigma_{x}^{(p)}\left[\sin \theta_{2}^{(p)}-\sin \theta_{1}^{(p)}\right]-\tau_{x y}^{(p)}\left[\cos \theta_{2}^{(p)}-\cos \theta_{1}^{(p)}\right]\right)=0, \\
\sum_{p=I}^{K}\left(\tau_{x y}^{(p)}\left[\sin \theta_{2}^{(p)}-\sin \theta_{1}^{(p)}\right]-\sigma_{y}^{(p)}\left[\cos \theta_{2}^{(p)}-\cos \theta_{1}^{(p)}\right]\right)=0,
\end{gathered}
$$

where $\theta_{2}^{(\mathrm{p})}$ and $\theta_{1}^{(p)}$ are starting and ending angles at joint for block $p$ as shown in Figure 12. 


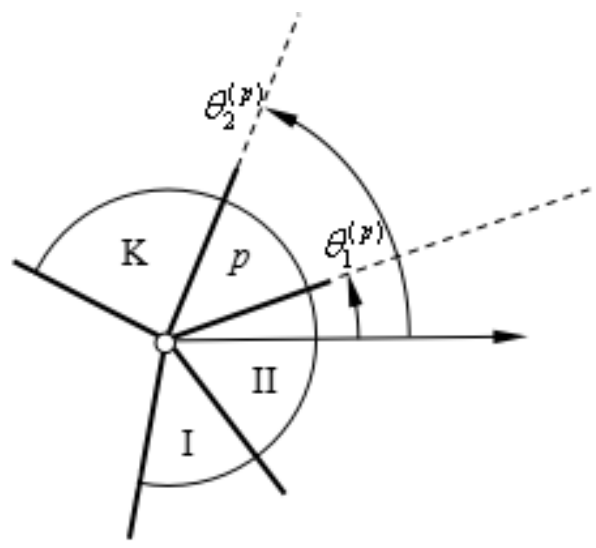

Figure 12: Corner joint relating to several blocks.

Due to the singularity at the crack tip, we calculate and compare two kinds of different distributions of seeds around this point. Case 1: the seeds A, B and C located at midpoint of each side with coordinates $(0.5 a, 0),(a, 0.5 h)$ and $(1.5 a, 0)$, respectively. Case 2: the seeds $\mathrm{A}, \mathrm{B}$ and $\mathrm{C}$ are shifted to $A^{\prime}(0.7 a, 0), B^{\prime}(a, 0.3 h)$ and $C^{\prime}(1.3 a, 0)$ which are closer to the crack-tip. The corresponding nodal distributions in physical region are given in Figure 13. When choosing material parameters $E_{1}=E_{2}=1$ unit, $v=0.3$ and $G=E_{1} / 2(1+v)$, the normal stress along the extension of the crack in block I and the vertical displacements at the crack surface in block II are presented in Figure 14 and 15, respectively. Numerical results calculated by BEM [38] with 64 boundary elements are shown also as a reference. It can be observe that results produced by irregularly distributed nodes are better than those by uniformly distributed ones.

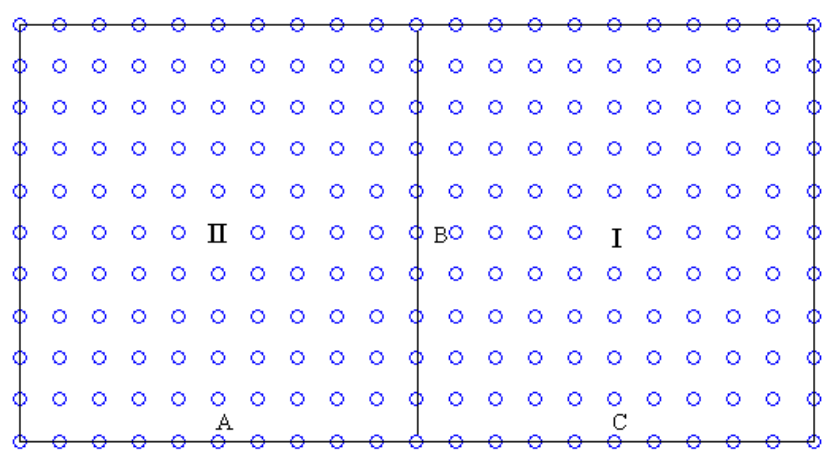


(a)

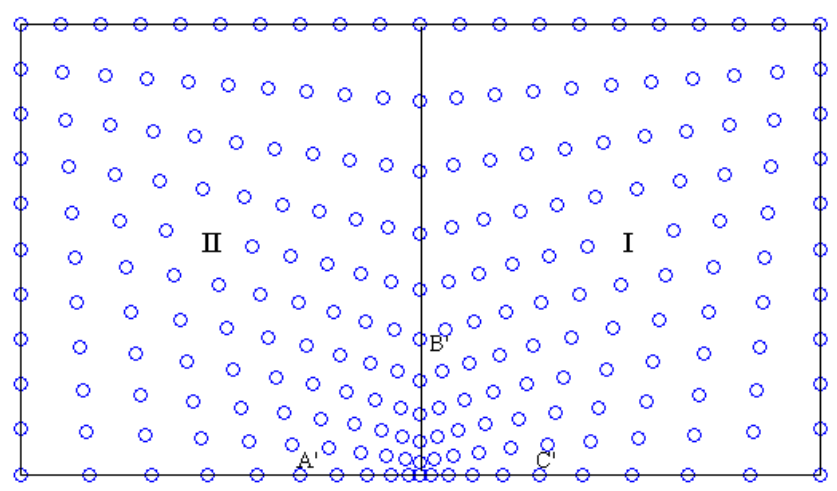

(b)

Figure 13: Nodal distribution in blocks for case 1 (a) and case 2 (b).

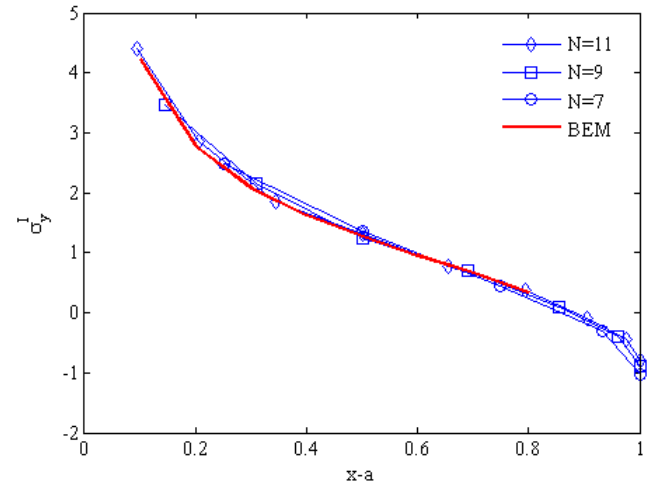

(a)

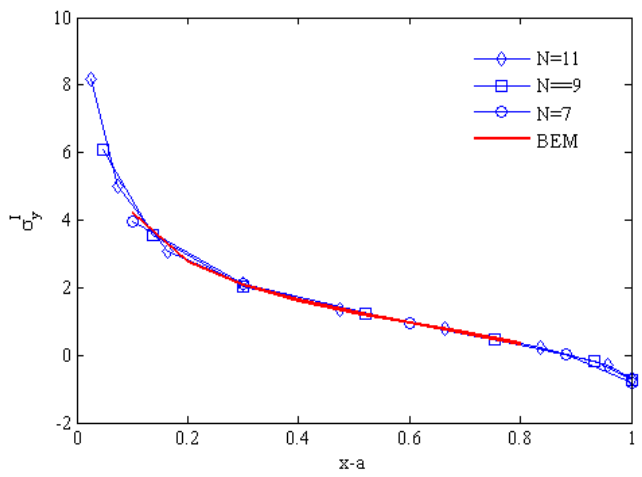

(b)

Figure 14: Numerical normal stresses along the extension of the crack in block I for case 1 (a) and case 2 (b).

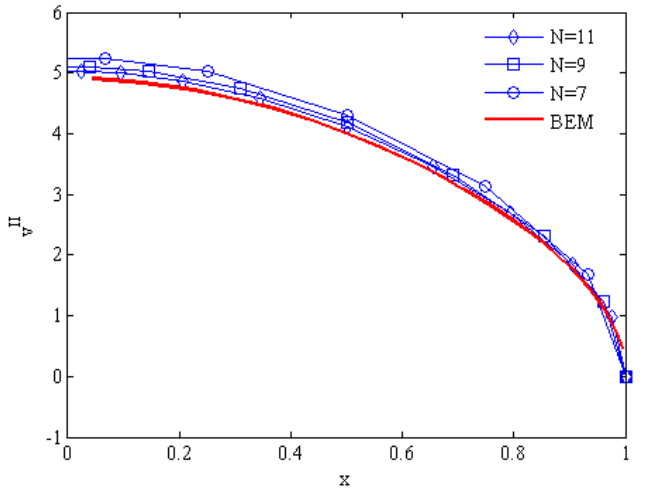

(a)

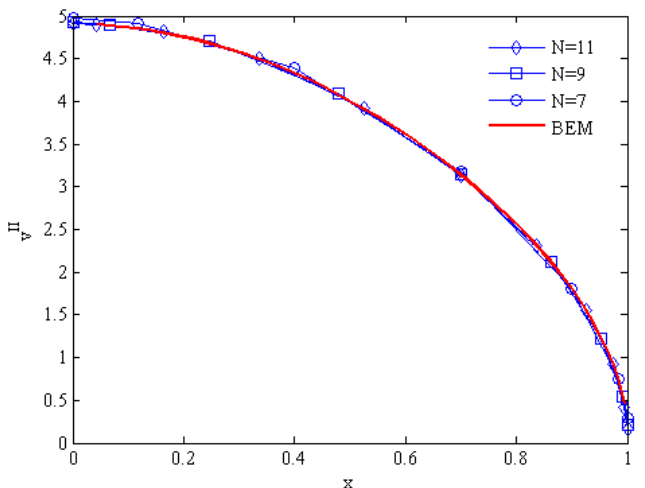

(b) 
Figure 15: Vertical displacements at the crack surface for case 1 (a) and case 2 (b).

Secondly, consider the plate with a central crack whose elastic modulus has a power law distribution as $E_{1}=E_{2}=\left(\sqrt{x^{2}+y^{2}}+1\right)^{2}$, other parameters are $v=0.3$ and $G=E_{1} / 2(1+v)$. Because the non-uniformly distributed nodes as shown in Figure 13(b) contribute to a better approximation, we adopt this distribution to compare numerical results calculated by the strong-form FBM and the weak-form BPGM. The normal stresses along the extension of the crack in block I and vertical displacements at the crack surface are plotted in Figure 16 and 17, respectively. Form which we can observe that the introduced BPGM works well for functionally graded materials with power law distribution also.

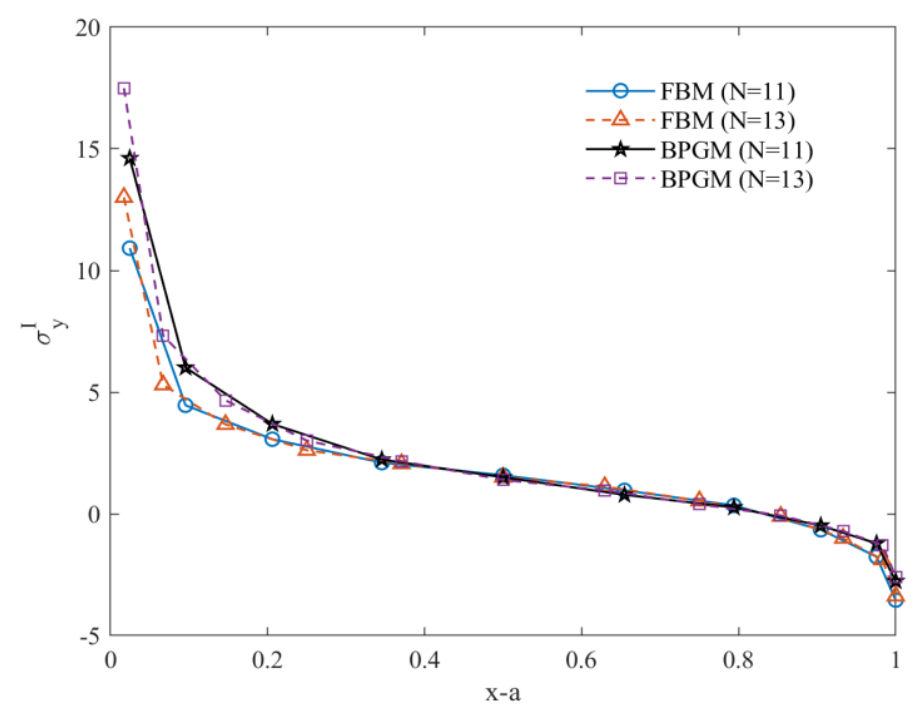

Figure 16: Comparison of normal stresses along the extension of the crack in block I. 


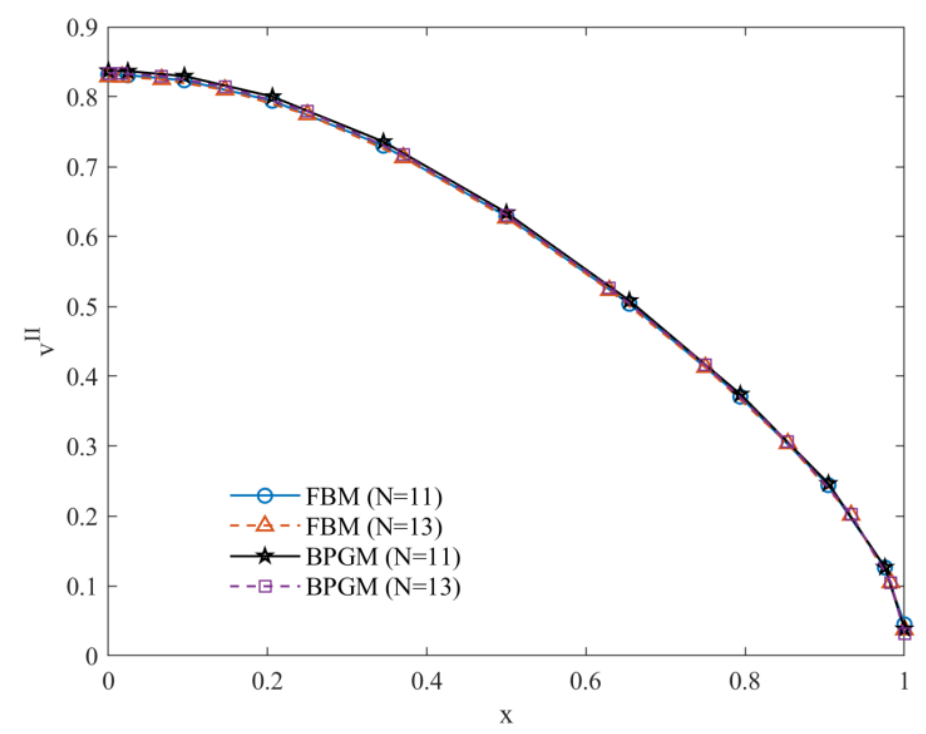

Figure 17: Comparison of vertical displacements at the crack surface.

Thirdly, a general case of cracks with isotropic functionally graded material is given to investigate the convergence of the proposed approach. The elastic modulus has an exponential variation relating to coordinates as $E_{1}=E_{2}=E_{0} e^{\left(\alpha_{1}|x|+\alpha_{2}|y|\right) / a}$, where $\alpha_{i}$ are constants and $E_{0}$ is the elastic modulus at the origin. The Poisson's ratio and shear modulus are the same as the previous example. When using different number of nodes $(N)$ on each dimension for interpolation, the normalized SIFs $K_{I} / \sigma_{0} \sqrt{\pi a}$ at the fourth node from the crack tip are presented in Table 2. They are calculated based on the crack opening displacement at the interface with formula:

$$
K_{I}=\frac{\sqrt{\pi a} \Delta u_{y}}{4 E_{t i p} \sqrt{2 r}}
$$

in which $\Delta u_{y}$ is the changing of vertical displacement at the crack interface, $E_{t i p}$ is Young's modulus at the crack tip and $r$ is the distance between the evaluation point and the crack tip. Numerical values agree well with the reference value (1.7677) given by FEM using 20000 quadratic shell elements via ABAQUS. Compared with results of strong-form FBM [40], the weak-form BPGM contributes to more stable solutions. Table 2: Normalized SIFs with different number of interpolation nodes on each dimension. 


\begin{tabular}{|c|c|c|c|c|c|c|c|}
\hline$N$ & 9 & 11 & 13 & 15 & 17 & 19 & 21 \\
\hline$K_{I} / \sigma_{0} \sqrt{\pi a}$ & 1.8321 & 1.8147 & 1.8077 & 1.8001 & 1.7948 & 1.7866 & 1.7856 \\
\hline
\end{tabular}

Example 6.3: Rectangular plate with an edge crack

In this example, we consider a strip with an edge crack of length $a$ unit in isotropic functionally graded media with width $w$ unit and height $2 h$ unit. Uniform tensile and bending loads are applied on the top of the plate while the bottom is fixed as shown in Figure 18. The material parameters are exponential functions relating to $x$ as: $E_{1}=E_{2}=E_{0} \exp (\alpha x / w)$, in which $\alpha=\ln \left(E_{w} / E_{0}\right), E_{0}$ and $E_{w}$ are Young's modulus on the left and right boundary of the strip, respectively. Other parameters are $v=0.3$ and $G=E_{1} / 2(1+v)$. For the sake of simplicity, the dimensions are normalized as $w=2 a, h=2 w$.

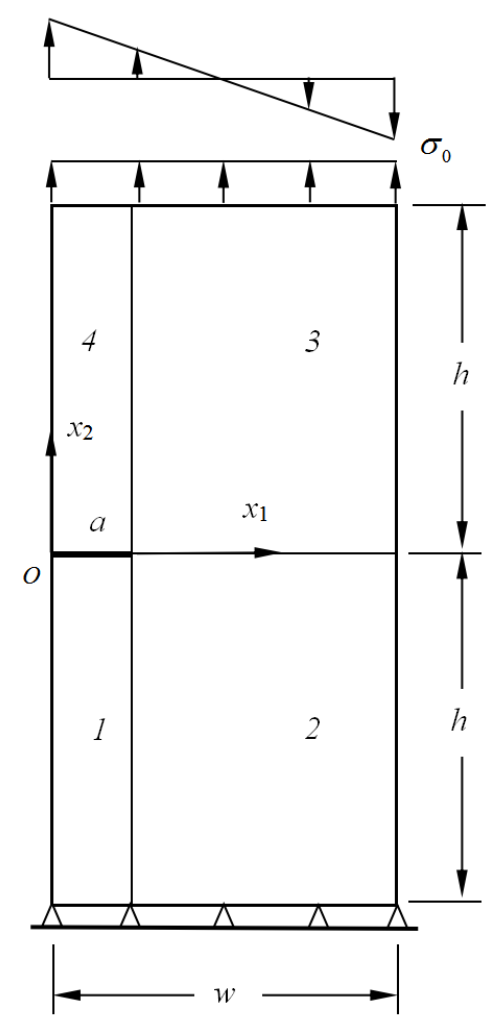

Figure 18: A plate with edge-crack subjected to tensile and bending loads.

For this problem, four finite blocks are employed and each one is covered by 
$N^{2}=21 \times 21$ nodes whose distribution are selected to be Chebyshev's roots as:

$$
\xi_{k}=\cos \frac{\pi(k-1)}{N-1}, \quad \eta_{l}=\cos \frac{\pi(l-1)}{N-1}, \quad k, l=1,2, \ldots, N .
$$

Table 3 and 4 present respectively numerical results of normalized SIFs $K_{I} / \sigma_{0} \sqrt{2 \pi a}$ subject to tensile and bending loads with respect to different ratios of material property $E_{w} / E_{0}$. For comparison, we give also the analytical values by Erdogan and $\mathrm{Wu}$ [44] and numerical solutions by FBM [42], FEM [43] and EFG [45-46]. Obviously, the BPGM produces a comparable accuracy with the existing efficient numerical approaches

Table 3: Normalized SIFs $K_{I} / \sigma_{0} \sqrt{\pi a}$ for edge-cracked strip subjected to tensile.

\begin{tabular}{|c|c|c|c|c|c|c|}
\hline$E_{w} / E_{0}$ & BPGM & $\begin{array}{c}\text { FBM } \\
{[40]}\end{array}$ & $\begin{array}{c}\text { Erdogan } \\
\text { and Wu } \\
{[42]}\end{array}$ & $\begin{array}{c}\text { Chen et al. } \\
{[43]}\end{array}$ & $\begin{array}{c}\text { Kim and } \\
\text { Paulino } \\
{[41]}\end{array}$ & $\begin{array}{c}\text { Rao and } \\
\text { Rahman } \\
{[44]}\end{array}$ \\
\hline 0.1 & 3.4310 & 3.4879 & 3.5701 & 3.5213 & 3.4960 & 3.5472 \\
\hline 0.2 & 3.2750 & 3.3224 & 3.3266 & 3.3234 & 3.2920 & 3.3297 \\
\hline 5 & 2.4294 & 2.4684 & 2.3656 & 2.4125 & 3.3660 & 2.3772 \\
\hline 10 & 2.2399 & 2.2826 & 2.1762 & 2.2234 & 2.1750 & 2.1889 \\
\hline
\end{tabular}

Table 4: Normalized SIFs $K_{I} / \sigma_{0} \sqrt{\pi a}$ for edge-cracked strip subjected to bending.

\begin{tabular}{|c|c|c|c|c|c|}
\hline$E_{w} / E_{0}$ & BPGM & $\begin{array}{c}\text { FBM } \\
{[40]}\end{array}$ & $\begin{array}{c}\text { Erdogan and } \\
\text { Wu [42] }\end{array}$ & $\begin{array}{c}\text { Kim and } \\
\text { Paulino [41] }\end{array}$ & $\begin{array}{c}\text { Rao and } \\
\text { Rahman [44] }\end{array}$ \\
\hline 0.1 & 2.2227 & 2.2669 & 2.2151 & 2.1450 & 2.1547 \\
\hline 0.2 & 2.0109 & 2.0408 & 1.9534 & 1.9250 & 1.9322 \\
\hline 5 & 1.2218 & 1.2366 & 1.1518 & 1.1580 & 1.1666 \\
\hline 10 & 1.0905 & 1.1066 & 1.0350 & 1.0350 & 1.0447 \\
\hline
\end{tabular}

\section{Example 6.4: Infinite strip containing a circular hole}

Consider an isotropic homogeneous infinite strip containing a circular hole with radius $R$ equal to $a$ unit under a uniaxial load $\sigma_{0}$, as shown in Figure 19. Due to symmetry of the problem, only a quarter of plate is modeled with two finite blocks and one infinite block. Because the edge of infinite block at reachable location is straight, all the mapping introduced in section 2.3 can be employed. In order to capture the stress concentration better, the coordinate of nodes in mapped domains are 
selected to be Chebyshev's roots as given in Eq.(38). The analytical solution of maximum stress at point $\mathrm{A}$ is $\sigma_{\max }=K \sigma_{0} h(h-a)$ [41], where $K$ is the stress concentration factor which can be expressed as

$$
K=3.00-3.13\left(\frac{a}{h}\right)+3.66\left(\frac{a}{h}\right)^{2}-1.53\left(\frac{a}{h}\right)^{3} .
$$

By using BPGM, numerical results of stress concentration factor versus the ratio of radius of the circular hole and half height of the strip are shown in Figure 20, which are in good agreement with analytical values.

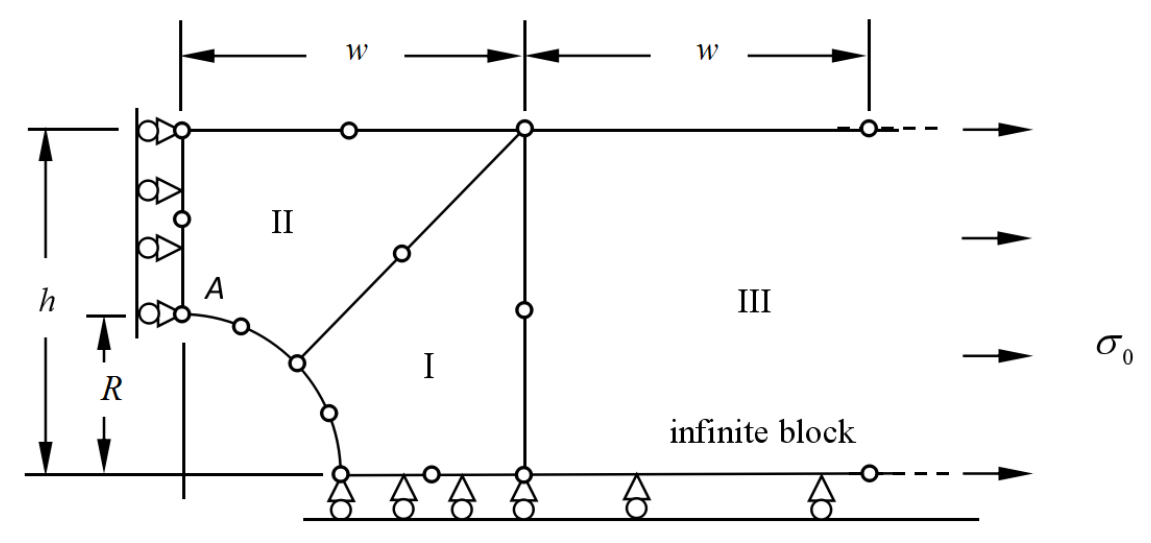

Figure 19: Blocks in a quarter of infinite strip containing a circular hole.

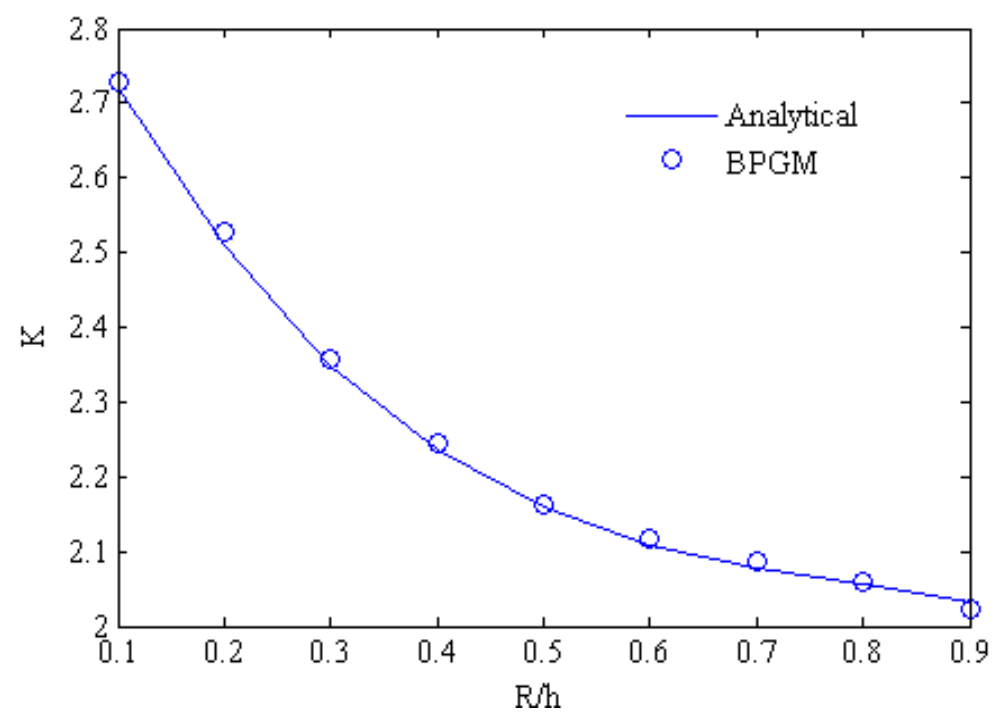

Figure 20: Stress concentration factor $K$ for a strip with circular hole.

Secondly, consider the same geometry with non-homogenous FGMs. The elastic 
modulus has an exponential variation in $y$-axis as $E_{1}=E_{2}=E_{0} e^{\alpha|y|}$, where $\alpha$ is arbitrary constant, $E_{0}$ is elastic modulus at the bottom and selected to be one unit here. It represents that an infinite strip bonded with two strips of FGM in engineering. The Poisson's ratio and shear modulus are the same as those in previous examples. When dealing with this unbounded problem with BPGM, it is necessary to carry out by the following two steps:

Step 1: Deduce the general solution of stresses by using the Saint-Venant's Principle, we have:

$$
\sigma_{x}^{0}=\sigma_{0}, \quad \sigma_{y}^{0}=0, \tau_{x y}^{0}=0
$$

Step 2: Find a particular solution $\sigma_{x}^{\prime}, \sigma_{y}^{\prime}$ and $\tau_{x y}^{\prime}$ by applying traction boundary condition on the hole as follows:

$$
t_{x}=-\sigma_{0} \cos \theta, t_{y}=0,
$$

where $\theta=a \tan (y / x)$. Then the numerical and shear stresses can be evaluated as: $\sigma_{x}=\sigma_{x}^{0}+\sigma_{x}^{\prime}, \sigma_{y}=\sigma_{y}^{0}+\sigma_{y}^{\prime}$ and $\tau_{x y}=\tau_{x y}^{0}+\tau_{x y}^{\prime}$. When $a / h=0.5$, Figure 21 and 22 present the distribution of normal stress along y-axis when $x=0$ for different $\alpha$. While comparing with reference values given by FEM (ABAQUS) using a large amount of elements, the BPGM calculated by a few blocks produces comparable solutions. 


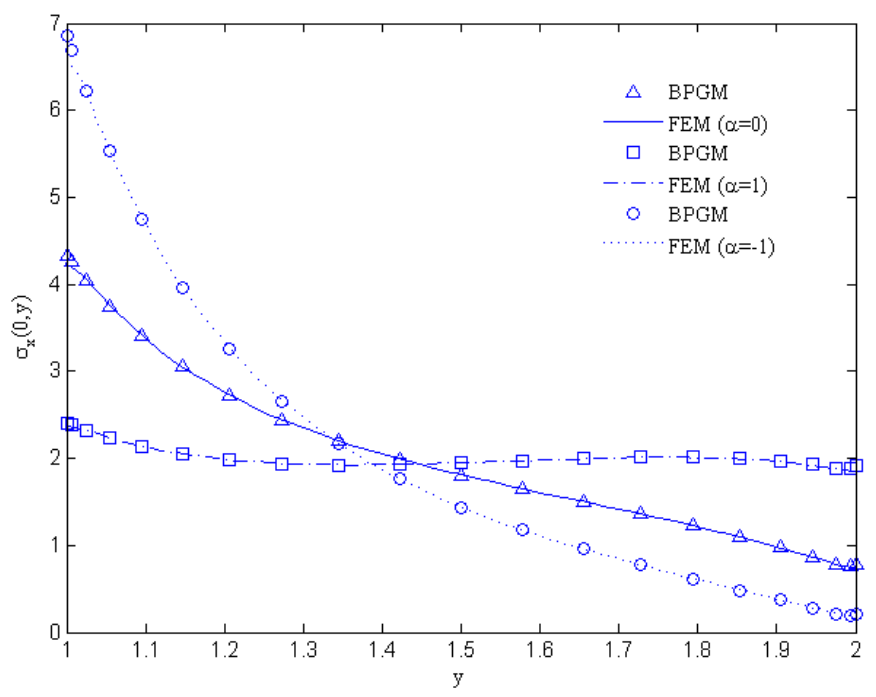

Figure 21: Normal stress $\sigma_{x}$ of points along $y$-axis when $x=0$.

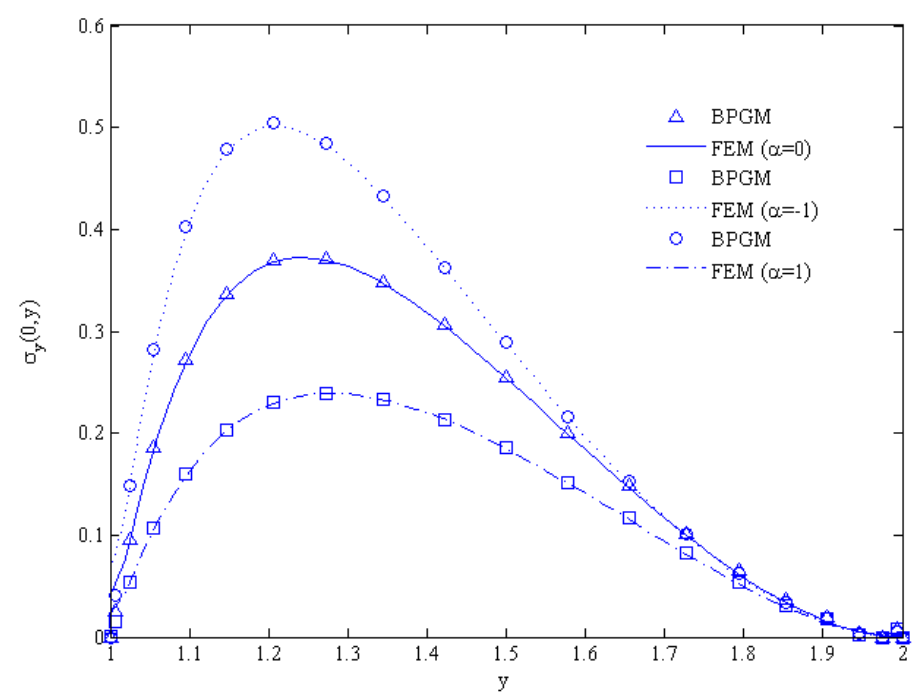

Figure 22: Normal stress $\sigma_{y}$ of points along $y$-axis when $x=0$.

\section{Example 6.5. Edge-cracked infinite strip under tension $\sigma_{0}$}

Finally we consider the edge-cracked infinite strip of height $2 h$ unit with a central crack of length $2 a$ unit subjected to a uniform tensile load $\sigma_{0}$ at infinity as shown in Figure 23. Four blocks including two finite blocks and two infinite blocks are required for calculation in this case. Chebyshev's nodal distributions (Eq.(49)) are adopted in each mapped domain with density of $N^{2}=21 \times 21$. The calculated normalized SIFs $K_{I} / \sigma_{0} \sqrt{\pi a}$ of the fourth node from the crack-tip are plotted in 
Figure 24, from which we observed that the biggest error occurs when $a / h=0.1$ and the relative error is around $5.8 \%$.

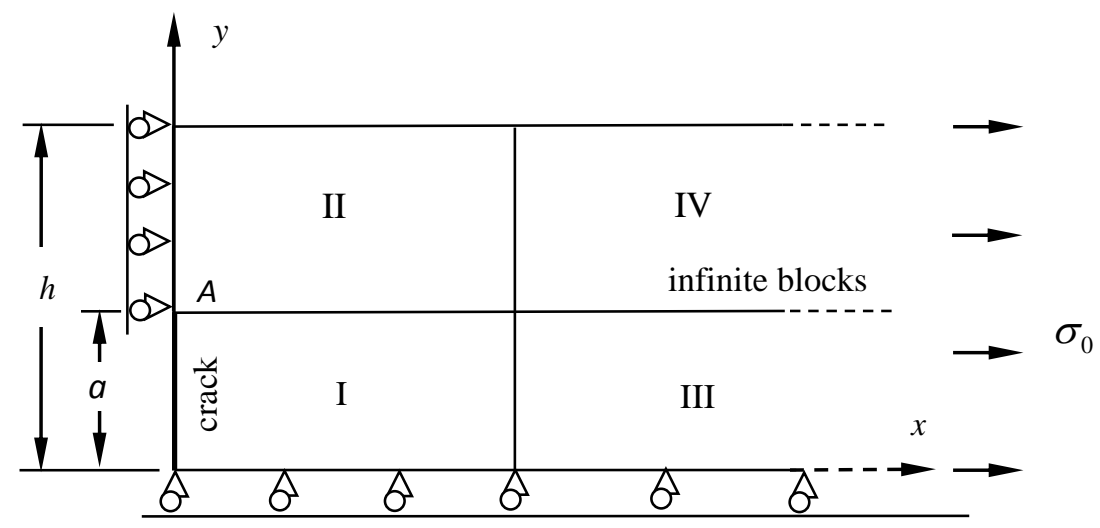

Figure 23: Edge-cracked infinite strip subjected to a tensile load.

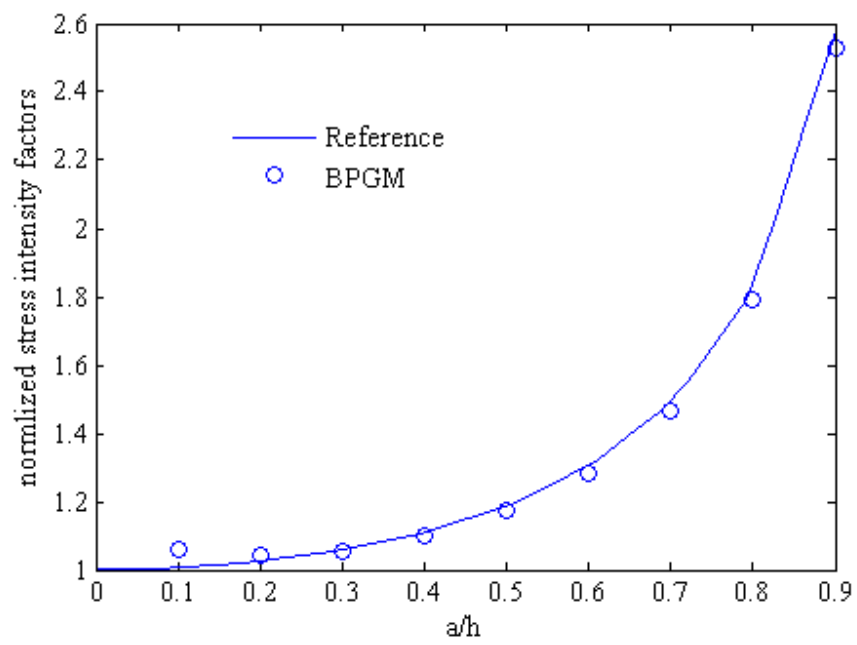

Figure 24: Normalized stress intensity factor versus the ratio of $a / h$.

\section{Conclusion}

The block Petrov-Galerkin method which employs finite/infinite blocks and combined with the meshless local Petrov-Galerkin method is proposed for solving two-dimensional linear elastic fracture mechanics with anisotropic functionally graded materials. Quadratic type of blocks with eight seeds are chosen for problems in finite field, while for problems in unbounded regions, infinite blocks with four-seed mapping, five-seed mapping and six-seed mapping can be selected. Once the physical domain is divided into blocks, which are mapped into normalized square domains, the meshless 
local Petrov-Galerkin method are employed with Lagrange series of interpolations in each block. The system equations are formulated by equilibrium equations, boundary conditions and continuous properties for all blocks. The stress intensity factors are obtained by crack opening displacement techniques. The essential features of the proposed approach and numerical results can be summarized as:

(1) The BPGM produces comparable results with the finite element analysis solutions and those of meshless approaches such as the BEM and EFG. Compared with FEM which requires a large amount of high-quality elements, the proposed method works with a few blocks reduce the calculation greatly. Because of using polynomials for interpolation in sub-regions, the precision is fine and there is no free parameters to determine.

(2) Due to the use of the weak-form Petrov-Galerkin method, the continuity requirements on the approximation functions are reduced and Neumann boundary conditions are satisfied naturally. Comparing with the existing strong-form FBM, numerical solutions produced by the weak-form block approach is more stable.

(3) The infinite blocks with quadratic shape functions are introduced for problems in unbounded field. This helps to avoid the negative effect of boundary conditions and reduce the computation cost.

(4) To model the elastic mechanics problems with cracks, it is necessary to discretized the domain into several blocks, especially at the tip of a crack. And continuous conditions of displacements and stresses should be considered at the interface of neighboring blocks.

(5) In addition, the distribution of nodes in normalized domains are selected to be the Chebyshev's roots for improving the accuracy.

However, apart from the above merits, the BPGM also has some limitations: (1) it is not a full-meshfree method and preprocess of block or domain discretization is still required; (2) it may consume additional computation for continuous conditions at the interface of neighboring blocks. 


\section{Reference}

[1] Mitchell AR, Griffiths DF. The finite difference method in partial differential equations. John Wiley, 1980.

[2] Zienkiewicz OC, Taylor RL, Zhu JZ. Finite Element Method: Its Basis and Fundamentals. Elsevier, Incorporated, 2013.

[3] Aliabadi MH. The boundary element method: applications in solids and structures. Chicester: Wiley, 2002.

[4] Liu GR. Mesh Free Methods Moving beyond the finite element Method. USA: CRC Press, 2003.

[5] Nayroles B, Touzot G, Villon P. Generalizing the finite element method: diffuse approximation and diffuse elements. Computational Mechanics. 1992, 10: 307-318.

[6] Belytschko T, Lu YY, Gu L. Element-free Galerkin method.International Journal of Numerical Methods in Engineering. 1994, 37: 229-256.

[7] Liu WK, Jun S, Zhang Y. Reproducing kernel particle methods. International Journal of Numerical Methods in Engineering. 1995, 20: 1081-1106.

[8] Atluri SN, Zhu T. A new meshless local Peyrov-Galerkin (MLPG) approach to nonlinear problems in computational modelling and simulation. Computer Modeling and Simulation in Engingering. 1998, 3: 187-196.

[9] Sladek J, Sladek V, Zhang CZ. Heat conduction analysis in nonhomogeneous anisotropic solid, ZH Yao, MW Yuan, WX Zhong edited. Computer Mechanics. Tsinghua University Press and Springer, 2004, 609-614.

[10]Golgerg MA, Chen CS, Karur SR. Improved multiquadric approximation for partial differential equations. Engineering Analysis with Boundary Elements. 1996, 18: 9-17.

[11]Hardy RL. Multiquadric equations of topography and other irregular surface. Journal of Geophysics Resources. 1971, 76: 1905-1915.

[12]Hon YC, Mao XZ. A multiquadric interpolation method for solving initial value problems. Journal of Scientific Computing. 1997, 12: 51-55.

[13] Sarler B, Vertnik R. Meshfree explicit local radial basis function collocation method for diffusion problems. Computer and Mathematical Application. 2006, 51(8): 1269-1282.

[14]Hon YC, Sarler B, Yun DF. Local radial basis function collocation method for solving thermo-driven fluid-flow problems with free surface. Engineering Analysis with Boundary Elements. 2015, 57: 2-8.

[15]Liu GR, Zhang GY. Smoothed Point Integration Methods: G Space theory and Weakened Weak Forms. World Scientific Publishing Co. Pte. Ltd, 2013.

[16]Li Y, Liu GR, Yue JH. A novel node-based smoothed radial point interpolation method for 2D and 3D solid mechanics problems. Computers \& Structures. 2018, 196: 157-172.

[17]Wen PH, Hon YC, Li M. Finite integration method for partial differential equations Applied Mathematical Modelling. 2013, 37(24): 10092-10106.

[18]Li Y, Hon YC. Finite integration method with radial basis function for solving 
stiff problems. Engineering Analysis with Boundary Elements 2017, 82: 32-42.

[19]Zienkiewicz OC, Kelly DW, Bettess P. The coupling of the finite element method and boundary solution procedures. International Journal for Numerical Methods in Engineering. 1977, 11(2): 355-375.

[20]Brebbia CA, Dominguez J. Boundary elements: an introductory course. WIT press, 1994.

[21] Wood WL. On the finite element solution of an exterior boundary value problem. International Journal of Numerical Methods in Engineering. 1976, 10: 885-891.

[22]Bettess P, Zienkiewicz OC. Diffraction and refraction of surface waves using finite and infinite elements. International Journal of Numerical Methods in Engineering. 1977; 11: 1271-1290.

[23] Khalili N, Valliappan S, Tabatabaie Yazdi J, Yazdchi M. 1D infinite element for dynamic problems in saturated porous media. Communications on Numerical Methods in Engineering. 1997, 13: 727-738.

[24]Bettess P. Infinite elements. International Journal of Numerical Methods in Engineering. 1977, 11: 53-64.

[25]Damjanic F, Owen DRJ. Mapped infinite element in transient thermal analysis. Computers and Structures. 1984, 19: 673-687.

[26]Zienkiewicz OC, Bando K, Bettess P, Emson C, Chiam TC. 1985. Mapped infinite element for exterior wave problems. International Journal of Numerical Methods in Engineering. 1985, 21: 1229-1251.

[27] Suresh S, Mortensen A. Fundamentals of functionally graded materials. The Institut of Materials, 1998.

[28] Paulino GH, Jin ZH, Dodds JRH. Failure of functionally graded materials. Comprehensive structural integrity. 2003, 2(13): 607-644.

[29]Erdogan F. Fracture mechanics of functionally graded materials. Composites Engineering, 1995, 5(7): 753-770.

[30] Sladek J, Sladek V, Zhang C. Evaluation of fracture parameters for crack problems in FGM by a meshless method. Journal of theoretical and applied mechanics. 2006, 44(3): 603-636.

[31]Noda N, Zhi-He J. Thermal stress intensity factors for a crack in a strip of a functionally gradient material. International Journal of Solids and Structures. 1993, 30(8): 1039-1056.

[32] Gu P, Asaro RJ. Cracks in functionally graded materials. International Journal of Solids and Structures. 1997, 34(1): 1-17.

[33] Ozturk M, Erdogan F. Mode I crack problem in an inhomogeneous orthotropic medium. International Journal of Engineering Science. 1997, 35(9): 869-883.

[34] Ozturk M, Erdogan F. The mixed mode crack problem in an inhomogeneous orthotropic medium. International Journal of Fracture. 1999, 98(3): 243-261.

[35] Kim JH, Paulino GH. The interaction integral for fracture of orthotropic functionally graded materials: evaluation of stress intensity factors. International Journal of Solids and Structures. 2003, 40(15): 3967-4001.

[36]Li M, Wen PH. Finite block method for transient heat conduction analysis in functionally graded media. International Journal for Numerical Methods in 
Engineering. 2014, 99(5): 372-390.

[37]Li M, Lei M, Munjiza A, Wen PH. Frictional contact analysis of functionally graded materials with Lagrange finite block method. International Journal for Numerical Methods in Engineering. 2015, 103(6): 391-412.

[38]Li M, Meng L X, Hinneh P, Wen PH. Finite block method for interface cracks. Engineering Fracture Mechanics. 2016, 156: 25-40.

[39]Li M, Monjiza A, Xu YG, Wen PH. Finite block Petrov-Galerkin method in transient heat conduction. Engineering Analysis with Boundary Elements. 2015, 60: 106-114.

[40]Wen PH, Cao P, Korakianitis T. Finite Block Method in elasticity. Engineering Analysis With Boundary Elements. 2014, 46: 116-125.

[41] Young WC, Budynas RG. Formulas for Stress and Strain. McGraw-Hill, New York,2002.

[42]Li J, Liu JZ, Korakianitis T, Wen PH. Finite block method in fracture analysis with functionally graded materials. Engineering Analysis with Boundary Elements. 2017, 82: 57-67.

[43] Kim JH, Paulino GH. Finite element evaluation of mixed mode stress intensity factors in functionally graded materials. International Journal for Numerical Methods in Engineering. 2002, 53(8): 1903-1935.

[44]Erdogan F and Wu BH. The surface crack problem for a plate with functionally graded properties. Journal of applied Mechanics. 1997, 64: 449.

[45]Chen J, Wu LZ, Du SY. Element-free Galerkin methods for fracture of functionally-graded materials. Key Engineering Materials. Trans Tech Publications. 2000, 183: 487-492.

[46]Rao BN, Rahman S. Mesh-free analysis of cracks in isotropic functionally graded materials. Engineering Fracture Mechanics. 2003, 70: 1-27.

\section{Appendix}

Partial differentials of shape functions with respect to normalized axes $\xi$ and $\eta$ for

eight-seed mapping:

$$
\begin{aligned}
& \frac{\partial N_{i}}{\partial \xi}=\frac{\xi_{i}}{4}\left(1+\eta_{i} \eta\right)\left(2 \xi_{i} \xi+\eta_{i} \eta\right), \\
& \frac{\partial N_{i}}{\partial \eta}=\frac{\eta_{i}}{4}\left(1+\xi_{i} \xi\right)\left(\xi_{i} \xi+2 \eta_{i} \eta\right), \text { for } i=1,2,3,4 \\
& \frac{\partial N_{i}}{\partial \xi}=-\xi\left(1+\eta_{i} \eta\right), \quad \frac{\partial N_{i}}{\partial \eta}=\frac{\eta_{i}}{2}\left(1-\xi^{2}\right), \text { for } i=5,7 \\
& \frac{\partial N_{i}}{\partial \xi}=\frac{\xi_{i}}{2}\left(1-\eta^{2}\right), \quad \frac{\partial N_{i}}{\partial \eta}=-\eta\left(1+\xi_{i} \xi\right), \text { for } i=6,8 .
\end{aligned}
$$


four-seed mapping:

$$
\begin{aligned}
& \frac{\partial N_{1}}{\partial \xi}=-\frac{(1-\eta)}{(1-\xi)^{2}}, \frac{\partial N_{2}}{\partial \xi}=\frac{(1-\eta)}{(1-\xi)^{2}}, \frac{\partial N_{3}}{\partial \xi}=\frac{(1+\eta)}{(1-\xi)^{2}}, \frac{\partial N_{4}}{\partial \xi}=-\frac{(1+\eta)}{(1-\xi)^{2}}, \\
& \frac{\partial N_{1}}{\partial \eta}=\frac{\xi}{(1-\xi)}, \frac{\partial N_{2}}{\partial \eta}=-\frac{(1+\xi)}{2(1-\xi)}, \frac{\partial N_{3}}{\partial \eta}=\frac{(1+\xi)}{2(1-\xi)}, \frac{\partial N_{4}}{\partial \eta}=-\frac{\xi}{(1-\xi)} .
\end{aligned}
$$

five-seed mapping:

$$
\begin{aligned}
& \frac{\partial N_{1}}{\partial \xi}=-\frac{(1-\eta)(2+\eta)}{(1-\xi)^{2}}, \frac{\partial N_{2}}{\partial \xi}=\frac{(1-\eta)}{(1-\xi)^{2}}, \frac{\partial N_{3}}{\partial \xi}=\frac{(1+\eta)}{(1-\xi)^{2}} \\
& \frac{\partial N_{4}}{\partial \xi}=-\frac{(1+\eta)(2-\eta)}{(1-\xi)^{2}}, \frac{\partial N_{5}}{\partial \xi}=\frac{2\left(1-\eta^{2}\right)}{(1-\xi)^{2}} \\
& \frac{\partial N_{1}}{\partial \eta}=\frac{(2 \eta+\xi)}{(1-\xi)}, \frac{\partial N_{2}}{\partial \eta}=-\frac{(1+\xi)}{2(1-\xi)}, \frac{\partial N_{3}}{\partial \eta}=\frac{(1+\xi)}{2(1-\xi)}, \frac{\partial N_{4}}{\partial \eta}=\frac{(2 \eta-\xi)}{(1-\xi)}, \frac{\partial N_{5}}{\partial \eta}=-\frac{4 \eta}{(1-\xi)}
\end{aligned}
$$

six-seed mapping:

$$
\begin{aligned}
& \frac{\partial N_{1}}{\partial \xi}=-\frac{\left(\eta^{2}-\eta\right)}{(1-\xi)^{2}}, \frac{\partial N_{2}}{\partial \xi}=\frac{\left(\eta^{2}-\eta\right)}{(1-\xi)^{2}}, \frac{\partial N_{3}}{\partial \xi}=\frac{\left(\eta^{2}+\eta\right)}{(1-\xi)^{2}}, \\
& \frac{\partial N_{4}}{\partial \xi}=-\frac{\left(\eta^{2}+\eta\right)}{(1-\xi)^{2}}, \frac{\partial N_{5}}{\partial \xi}=-\frac{2\left(1-\eta^{2}\right)}{(1-\xi)^{2}}, \frac{\partial N_{6}}{\partial \xi}=\frac{2\left(1-\eta^{2}\right)}{(1-\xi)^{2}}, \\
& \frac{\partial N_{1}}{\partial \eta}=-\frac{\xi(2 \eta-1)}{(1-\xi)}, \frac{\partial N_{2}}{\partial \eta}=\frac{(1+\xi)(2 \eta-1)}{2(1-\xi)}, \frac{\partial N_{3}}{\partial \eta}=\frac{(1+\xi)(2 \eta+1)}{2(1-\xi)}, \\
& \frac{\partial N_{4}}{\partial \eta}=-\frac{\xi(2 \eta+1)}{(1-\xi)}, \frac{\partial N_{5}}{\partial \eta}=\frac{4 \xi \eta}{(1-\xi)}, \frac{\partial N_{6}}{\partial \eta}=-\frac{2(1+\xi) \eta}{(1-\xi)}
\end{aligned}
$$

